\title{
Homoclinic orbits for an unbounded superquadratic
}

\author{
Jun Wang, Junxiang Xu, Fubao Zhang and Lei Wang
}

\begin{abstract}
We consider the following nonperiodic diffusion systems $\left\{\begin{array}{l}\partial_{t} u-\triangle_{x} u+b(t, x) \nabla_{x} u+V(x) u=G_{v}(t, x, u, v), \\ -\partial_{t} v-\triangle_{x} v-b(t, x) \nabla_{x} v+V(x) v=G_{u}(t, x, u, v),\end{array} \quad \forall(t, x) \in \mathbb{R} \times \mathbb{R}^{N}\right.$, where $b \in C\left(\mathbb{R} \times \mathbb{R}^{N}, \mathbb{R}^{N}\right), G \in C^{1}\left(\mathbb{R} \times \mathbb{R}^{N} \times \mathbb{R}^{2 m}, \mathbb{R}\right)$ and $z:=(u, v)$ : $\mathbb{R} \times \mathbb{R}^{N} \rightarrow \mathbb{R}^{m} \times \mathbb{R}^{m}$. Suppose that the potential $\mathrm{V}$ is positive constant and $G(t, x, z)$ is superquadratic in $z$ as $|z| \rightarrow \infty$. By applying a generalized linking theorem for strongly indefinite functionals, we obtain homoclinic solutions $z$ satisfying $z(t, x) \rightarrow 0$ as $|(t, x)| \rightarrow \infty$.
\end{abstract}

Mathematics Subject Classification (2000). 58E50 (Variational problems in infinite-dimensional spaces, Applications).

Keywords. Unbounded Hamiltonian systems . Variational methods . $(C)_{c}$-condition.

\section{Introduction and main results}

\subsection{The problem}

In this paper we study the existence of homoclinic solutions for the system

$$
\left\{\begin{array}{l}
\partial_{t} u-\triangle_{x} u+b(t, x) \nabla_{x} u+V(x) u=G_{v}(t, x, u, v), \\
-\partial_{t} v-\triangle_{x} v-b(t, x) \nabla_{x} v+V(x) v=G_{u}(t, x, u, v),
\end{array} \quad \forall(t, x) \in \mathbb{R} \times \mathbb{R}^{N} .\right.
$$

Here $V \in C\left(\mathbb{R}^{N}, \mathbb{R}\right), b:=\left(b_{1}, \ldots, b_{N}\right) \in C\left(\mathbb{R} \times \mathbb{R}^{N}, \mathbb{R}^{N}\right)$ and $G \in C^{1}\left(\mathbb{R} \times \mathbb{R}^{N} \times\right.$ $\left.\mathbb{R}^{2 m}, \mathbb{R}\right), z:=(u, v): \mathbb{R} \times \mathbb{R}^{N} \rightarrow \mathbb{R}^{m} \times \mathbb{R}^{m}$. Such problems arise in optimal control of systems governed by partial differential equations (cf. Lions [27]) and are related to the Schrödinger equations (cf. Nagasawa [30]). If we let

$$
\mathcal{J}_{0}=\left(\begin{array}{cc}
0 & I \\
I & 0
\end{array}\right), \quad \mathcal{J}=\left(\begin{array}{cc}
0 & -I \\
I & 0
\end{array}\right), \quad \mathcal{S}:=-\triangle_{x}+V
$$

This work was supported by the Natural Science Foundation of China and the Scientific Research Foundation of Graduate School of Southeast University(YBJJ0928). 
and

$$
A=\mathcal{J}_{0} \mathcal{S}+\mathcal{J} b \cdot \nabla_{x},
$$

then the system $(H S)$ can be rewritten as

$$
\mathcal{J} \frac{d}{d t} z+A z=G_{z}(t, x, z)
$$

Thus the system $(H S)$ can be regarded as an unbounded Hamiltonian system (cf. Barbu [7]), or an infinite-dimensional Hamiltonian system in $L^{2}(\mathbb{R} \times$ $\left.\mathbb{R}^{N}, \mathbb{R}^{2 m}\right)$ (cf. $\left.[4,16,17]\right)$. In this paper, we are interested in the case that

$$
G(t, x, z)=-\frac{1}{2} B(t, x) z \cdot z+H(t, x, z),
$$

where $B(t, x)$ is $2 m \times 2 m$ symmetric bounded value matrix and $H(t, x, z)$ is superquadratic in $z$ as $|z| \rightarrow \infty$.

For the case of bounded domain, if $b \equiv 0$ and $V \equiv 0$, there are a number of papers considering the stationary solution of the system

$$
\begin{cases}-\triangle u=F_{v}(x, u, v) & \text { in } \Omega \subset \mathbb{R}^{N}, \\ -\triangle v=F_{u}(x, u, v) & \text { in } \Omega, \\ u(x)=v(x)=0 & \text { on } \partial \Omega .\end{cases}
$$

For example, see $[12,13,19,23,24,28,29]$ and the references therein. Particularly, in the interesting paper [29], the authors considered the case that $F(u, v)=\lambda|u|^{2}+|u|^{p+1}+\mu|v|^{2}+|v|^{q+1}$, where $\lambda, \mu$ are real numbers, and $\frac{1}{p+1}+\frac{1}{q+1} \leq \frac{N-2}{N}(N \geq 4)$. If $\lambda=\mu, p=q$ and $u=v$, the $\operatorname{system}(\mathcal{S})$ reduces to the scalar semilinear elliptic equation $-\Delta u=\lambda u+|u|^{p-1} u$ with critical exponent $p=\frac{N+2}{N-2}$. This leads to the lack of compactness for the corresponding critical functional. In order to circumvent this difficulty, they reduce the strongly indefinite critical functional to the mountain pass geometric case. Recently, De Figueiredo and Ding [20] studied the system

$$
\begin{cases}-\triangle u=H_{u}(x, u, v) & \text { in } \Omega \subset \mathbb{R}^{N}, \\ -\triangle v=-H_{v}(x, u, v) & \text { in } \Omega, \\ u(x)=v(x)=0 & \text { on } \partial \Omega,\end{cases}
$$

where $H$ satisfies $H(x, u, v) \sim|u|^{p}+|v|^{q}+R(x, u, v)$ with $\lim _{|u|+|v| \rightarrow \infty} \frac{R(x, u, v)}{|u|^{p}+|v|^{q}}$ $=0,1<p<2^{*}=\frac{2 N}{N-2}$ and $q>1$. They proved the existence of solutions for the above system. More recently, de Figueiredo, do Ó and Ruf [21] treated the following autonomous system via an Orlicz space approach

$$
\left\{\begin{array}{lll}
-\triangle u=g(v) \quad u(x)>0 & \text { in } \Omega, \\
-\triangle v=f(u) & v(x)>0 & \text { in } \Omega, \\
u(x)=v(x)=0, & \text { on } \partial \Omega .
\end{array}\right.
$$

Under some superlinear conditions on $f$ and $g$, the authors obtained one solution for the system. There is not much work on nonstationary solutions of 
systems like $(H S)$. Brézis and Nirenberg [8] considered the following parabolic system

$$
\left\{\begin{array}{l}
\partial_{t} u-\triangle_{x} u=-v^{5}+f \\
-\partial_{t} v-\triangle_{x} v=u^{3}+g
\end{array} \quad \forall(t, x) \in(0, T) \times \Omega,\right.
$$

where $f, g \in L^{\infty}$ and $\Omega$ is a smoothly bounded domain in $\mathbb{R}^{N}$. Moreover, $u$ and $v$ subject to the boundary conditions $u=v=0$ on $(0, T) \times \partial \Omega$ and $u(0, x)=v(T, x)=0$ on $\Omega$. Using Schauder's fixed point theorem they obtained a solution $(u, v)$ with $u \in L^{4}$ and $v \in L^{6}$. Clément et al. [11] investigated the following system

$$
\begin{cases}\partial_{t} u-\triangle_{x} u=|v|^{p-1} v & \text { in } \mathbb{R} \times \Omega, \\ -\partial_{t} v-\triangle_{x} v=|u|^{q-1} u & \text { in } \mathbb{R} \times \Omega, \\ \left.u(t, \cdot)\right|_{\partial \Omega}=\left.v(t, \cdot)\right|_{\partial \Omega}=0 & \text { on } \mathbb{R} \times \Omega,\end{cases}
$$

where $\Omega$ is a smoothly bounded domain in $\mathbb{R}^{N}$ and $N /(N+2)<1 / p+1 / q<1$. By using a mountain pass argument, they proved that there exists $T_{0}>0$ such that for each $T>T_{0}$, the system $(\mathcal{J})$ has at least one positive solution. Moreover, by passing to the limit as $T \rightarrow \infty$, they showed that the system $(\mathcal{J})$ has at least one positive solution satisfying

$$
\lim _{|t| \rightarrow \infty} u(t, x)=0=\lim _{|t| \rightarrow \infty} v(t, x) \quad \text { uniformly in } x \in \Omega .
$$

In the paper [10], Clément and Van der Vorst discussed the optimality of the condition $N /(N+2)<1 / p+1 / q<1$ on star-shaped domain.

Up to now, there are several authors considering this problem in $\mathbb{R}^{N}$. Suppose $b \equiv 0$ and $V \equiv 1, \mathrm{G}$ is radial in $x$ and is even in $z$. Bartsch and De Figueiredo [9] proved that the system, without the depending on time variable, admits infinitely many radial as well as non-radial stationary solutions. For periodic setting, assuming $b \equiv 0$, and under some conditions on $G$, Bartsch and Ding [4] established a proper variational framework and proved that the system has infinitely many homoclinic orbits. Their results were later generalized by Schecter and Zou [31]. Nearly, Ding et al. [16] considered $(H S)$ with $b(t, x) \not \equiv 0$ via variational methods. Under some periodic assumptions on $V(x), b(t, x)$ and $G(t, x, z)$, the existence of infinitely many solutions were obtained in their paper [16] for both superquadratic or asymptotically linear cases. For nonperiodic case, there has not much works done up to know. More recently, Zhao and Ding [32] considered the nonperiodic asymptotically linear case, and they proved the existence and multiplicity of solutions for $(H S)$. Up to present, an interesting problem of existence of homoclinic orbits for $(H S)$ with the nonperiodic superquadratic nonlinearity is still left open. Motivated by the present works, in this paper, we first devote to study such a case. The main difficulty here is the lack of compactness of the Sobolev's embedding, since the domain is the whole space. In order to overcome this difficulty, we use the methods which was used in $[1,14,15,29]$ to reduce the strongly indefinite critical functional to the mountain pass geometric case. Therefore, we can 
construct a linking level for the energy functional, and show that it has the local compactness.

For stating our main results, we first give some notations and definitions. For two given $2 m \times 2 m$ symmetric real matrix functions $L_{0}(t, x)$ and $L_{2}(t, x)$, we write $L_{0}(t, x)<L_{2}(t, x)$ if and only if

$$
\max _{\xi \in \mathbb{R}^{2 m}|\xi|=1}\left(L_{0}(t, x)-L_{2}(t, x) \xi \cdot \xi<0 .\right.
$$

Define similarly $L_{0}(t, x) \leq L_{2}(t, x)$. We denote $B(\infty):=\lim _{|(t, x)| \rightarrow \infty} B(t, x)$ when it exists. Set $\lambda_{1}=\max \{\lambda>0: \lambda \in \sigma(B(\infty))\}$ and $\lambda_{2}=\min \{\lambda<0$ : $\lambda \in \sigma(B(\infty))\}$, where $\sigma(B(\infty))$ denotes the eigenvalues of the matrix $B(\infty)$. Let $\tilde{H}(t, x, z):=\frac{1}{2} H_{z}(t, x, z) z-H(t, x, z)$, we assume further on $b(t, x), V$ and $H(t, x, z)$ that the following conditions are satisfied:

$\left(\mathcal{V}_{0}\right) V(x) \equiv V_{0}>0$ and $b(t, x)$ is a constant vector, i.e. $b(t, x) \equiv b_{0}$;

$\left(\mathcal{H}_{0}\right) B(\infty)$ exists, $B(t, x) \leq B(\infty)$ and $B_{L}:=B(\infty)-B(t, x)$ such that

$$
0<\lambda=\max _{(t, x) \in \mathbb{R} \times \mathbb{R}^{N}} \max _{|\xi|=1}\left(B_{L}\right) \xi \cdot \xi<V_{0}-\mu_{\infty}
$$

where $\mu_{\infty}:=\max \left\{\lambda_{1},\left|\lambda_{2}\right|\right\}$

$\left(\mathcal{H}_{1}\right) H(t, x, z) \in C^{1}\left(\mathbb{R} \times \mathbb{R}^{N} \times \mathbb{R}^{2 m}, \mathbb{R}\right), H(t, x, z) \geq 0$ and $H_{z}(t, x, z)=$ $o(|z|)$ as $|z| \rightarrow 0$ uniformly in $(t, x)$;

$\left(\mathcal{H}_{2}\right) H(t, x, z)|z|^{-2} \rightarrow \infty$ as $|z| \rightarrow \infty$ uniformly in $(t, x)$;

$\left(\mathcal{H}_{3}\right) \inf _{(t, x) \in \mathbb{R} \times \mathbb{R}^{N}} \tilde{H}(t, x, z)>0$ if $z \neq 0$, and there exist $r_{1}>0, c_{1}>0$, $\mu>1$ if $N=1, \mu>1+\frac{N}{2}$ if $N \geq 2$ such that $\left|H_{z}(t, x, z)\right|^{\mu} \leq c_{1} H(t, x, z)|z|^{\mu}$ if $|z| \geq r_{1}$;

$\left(\mathcal{H}_{4}\right)$ There exists $H_{\infty}(z) \in C^{2}\left(\mathbb{R}^{2 m}, \mathbb{R}\right)$ with $H_{\infty}(0)=0$ and $H_{\infty}^{\prime}(z)=$ $o(|z|)$ as $|z| \rightarrow 0$ such that $H(t, x, z) \geq H_{\infty}(z)$ for all $(t, x) \in \mathbb{R} \times \mathbb{R}^{N}$, and

(i) $H_{z}(t, x, z) \rightarrow H_{\infty}^{\prime}(z)$ as $|(t, x)| \rightarrow \infty$ uniformly in bounded set of $z$;

(ii) $H_{\infty}^{\prime}(w)(w+2 y) \leq H_{\infty}^{\prime \prime}(w)(w+y) \cdot(w+y)$ for all $w, y \in \mathbb{R}^{2 m}$.

Here, $H_{\infty}^{\prime}(z):=\nabla_{z} H_{\infty}(z), H_{\infty}^{\prime \prime}(z):=\triangle_{z} H_{\infty}(z)$ and $\tilde{H}_{\infty}(z):=\frac{1}{2} H_{\infty}^{\prime}(z) z-$ $H_{\infty}(z)$.

As in [4], we introduce the anisotropic space

$$
\begin{aligned}
B_{r} & :=B_{r}\left(\mathbb{R} \times \mathbb{R}^{N}, \mathbb{R}^{2 m}\right) \\
& :=W^{1, r}\left(\mathbb{R}, L^{r}\left(\mathbb{R}^{N}, \mathbb{R}^{2 m}\right)\right) \cap L^{r}\left(\mathbb{R}, W^{2, r}\left(\mathbb{R}^{N}, \mathbb{R}^{2 m}\right) \cap W_{0}^{1, r}\left(\mathbb{R}^{N}, \mathbb{R}^{2 m}\right)\right)
\end{aligned}
$$

equipped with the norm

$$
\|z\|_{B_{r}}:=\left(\int_{\mathbb{R} \times \mathbb{R}^{N}}\left(|z|^{r}+\left|\partial_{t} z\right|^{r}+\sum_{j=1}^{N}\left|\partial_{x_{j}}^{2} z\right|^{r}\right)\right)^{\frac{1}{r}},
$$

where $r \geq 1$. It is easy to see that $B_{r}$ is the completion of $C_{0}^{\infty}\left(\mathbb{R} \times \mathbb{R}^{N}, \mathbb{R}^{2 m}\right)$ with respect to the norm $\|\cdot\|_{B_{r}}$. Moreover, if $r=2, B_{2}$ is a Hilbert space.

For a solution $z$ of $(H S)$, we denote the associated action integral by

$$
\Phi(z):=\int_{\mathbb{R} \times \mathbb{R}^{N}}\left[\frac{1}{2}\left(\mathcal{J} \frac{d}{d t} z \cdot z+A z \cdot z\right)-G(t, x, z)\right] .
$$


Set

$$
c_{\text {min }}:=\inf \{\Phi(z) \mid z \neq 0 \text { is a solution (HS) }\} .
$$

A solution $z_{0} \neq 0$ with $\Phi\left(z_{0}\right)=c_{\min }$ is said to be a least action solution. Let $S_{\text {min }}$ denote the set of all least action solutions of $(H S)$.

Theorem 1.1. Let $\left(\mathcal{V}_{0}\right),\left(\mathcal{H}_{0}\right)-\left(\mathcal{H}_{4}\right)$ be satisfied. Then

(i) $(H S)$ has at least one nontrivial homoclinic orbit;

(ii) $S_{\text {min }}$ is compact in $B_{2}\left(\mathbb{R} \times \mathbb{R}^{N}, \mathbb{R}^{2 m}\right)$.

Remark 1.1. Let $\hat{x}=(t, x)$. A simple example of matrix functions satisfying $\left(\mathcal{H}_{0}\right)$ is the matrix function

$$
B(\hat{x})=\left(\begin{array}{cc}
\alpha(\hat{x}) & 0 \\
0 & -\beta(\hat{x})
\end{array}\right),
$$

where $\alpha(\hat{x})$ and $\beta(\hat{x})$ such that $\alpha_{0}=\lim _{|\hat{x}| \rightarrow \infty} \alpha(\hat{x})$ and $\beta_{0}=\lim _{|\hat{x}| \rightarrow \infty} \beta(\hat{x})$ exist, and $\max \left\{\left|\alpha_{0}\right|,\left|\beta_{0}\right|\right\}<V_{0}$ and

$$
\begin{aligned}
0 & \leq \alpha_{0}-\alpha(\hat{x})<\min \left\{V_{0}-\left|\alpha_{0}\right|, V_{0}-\left|\beta_{0}\right|\right\}, \\
0 \leq \beta(\hat{x})-\beta_{0} & <\min \left\{V_{0}-\left|\alpha_{0}\right|, V_{0}-\left|\beta_{0}\right|\right\}
\end{aligned}
$$

for all $\hat{x}$.

Remark 1.2. The following function satisfies $\left(\mathcal{H}_{1}\right)-\left(\mathcal{H}_{4}\right)$.

$\operatorname{Ex1.} H(t, x, z)=\frac{1}{2} a(t, x)\left(\left(|z|^{2}-1\right) \ln (1+|z|)+\frac{|z|}{2}(2-|z|)\right)$, where $a(t, x)>$ $0, a_{0}:=\lim _{|(t, x)| \rightarrow \infty} a(t, x)$ exists and $a(t, x) \geq a_{0}$ for all $(t, x)$.

\section{Preliminary works}

\subsection{Variational setting}

In this section, we first study the spectrum of the operator $L:=\mathcal{J} \partial_{t}+A$, where $A=\mathcal{J}_{0} \mathcal{S}+\mathcal{J} b \cdot \nabla_{x}$. By Lemma 2.2 of [32], we know that $L$ is a self-adjoint operator in $\mathcal{H}:=L^{2}\left(\mathbb{R} \times \mathbb{R}^{N}, \mathbb{R}^{2 m}\right)$ with the domain $\mathcal{D}(L)=B_{2}\left(\mathbb{R} \times \mathbb{R}^{N}, \mathbb{R}^{2 m}\right)$. Set $L_{B}:=L+B(t, x)$ and $L_{\infty}:=L+B(\infty)$, then by $\left(\mathcal{H}_{0}\right)$, we know that $\mathcal{D}(L)=\mathcal{D}\left(L_{\infty}\right)=\mathcal{D}\left(L_{B}\right)=B_{2}$. Let $\sigma_{d}(K), \sigma_{e}(K)$ denote, respectively, the discrete spectrum and the essential spectrum of the operator $K$. Let $|\cdot|_{q}$ denote the usual $L^{q}\left(\mathbb{R} \times \mathbb{R}^{N}, \mathbb{R}^{2 m}\right)$-norm and $(\cdot, \cdot)_{2}$ is the usual $L^{2}\left(\mathbb{R} \times \mathbb{R}^{N}, \mathbb{R}^{2 m}\right)$-inner product, $c$ or $c_{i}$ stands for different positive constants. As a special case of Lemma 2.1 of [16], we have

Lemma 2.1. Let $\left(\mathcal{V}_{0}\right)$ be satisfied. Then

(i) $\sigma(L)=\sigma_{e}(L)$, i.e., $L$ has only essential spectrum;

(ii) $\sigma(L) \subset \mathbb{R} \backslash\left(-V_{0}, V_{0}\right)$;

(iii) $\sigma(L)$ is symmetric with respect to origin, that is, $\sigma(L) \cap(0, \infty)=-\sigma(L) \cap$ $(-\infty, 0)$

(iv) $\quad \underline{\lambda}:=\inf \left\{\sigma_{e}(L) \cap(0, \infty)\right\}=V_{0}$.

Lemma 2.2. Under the conditions $\left(\mathcal{V}_{0}\right)$ and $\left(\mathcal{H}_{0}\right)$, one has

(i) $\sigma\left(L_{\infty}\right) \subset \mathbb{R} \backslash\left(-\left(V_{0}-\mu_{\infty}\right),\left(V_{0}-\mu_{\infty}\right)\right)$; 
(ii) $\sigma\left(L_{B}\right) \subset \mathbb{R} \backslash\left(-\left(V_{0}-\mu_{\infty}-\lambda\right),\left(V_{0}-\mu_{\infty}-\lambda\right)\right)$.

Proof. (i) By (ii) of Lemma 2.1, we have

$$
\begin{aligned}
\left|L_{\infty} z\right|_{2} & =\left|\left(L+B_{\infty}\right) z\right|_{2} \geq|L z|_{2}-|B(\infty) z|_{2} \\
& \geq V_{0}|z|_{2}-\mu_{\infty}|z|_{2}=\left(V_{0}-\mu_{\infty}\right)|z|_{2},
\end{aligned}
$$

thus $\sigma\left(L_{\infty}\right) \subset \mathbb{R} \backslash\left(-\left(V_{0}-\mu_{\infty}\right),\left(V_{0}-\mu_{\infty}\right)\right)$.

(ii) Similarly, since $L_{\infty}=L_{B}+(B(\infty)-B(t, x))$, then one has

$$
\begin{aligned}
\left|L_{B} z\right|_{2} & =\left|\left(L_{\infty}+(B(t, x)-B(\infty))\right) z\right|_{2} \geq\left|L_{\infty} z\right|_{2}-|(B(t, x)-B(\infty)) z|_{2} \\
& \geq\left(V_{0}-\mu_{\infty}\right)|z|_{2}-\lambda|z|_{2}=\left(V_{0}-\mu_{\infty}-\lambda\right)|z|_{2} .
\end{aligned}
$$

Thus we have $\sigma\left(L_{B}\right) \subset \mathbb{R} \backslash\left(-\left(V_{0}-\mu_{\infty}-\lambda\right),\left(V_{0}-\mu_{\infty}-\lambda\right)\right)$.

Now we consider the operator $L_{0}:=\mathcal{J}\left(\partial_{t}+b_{0} \cdot \nabla_{x}\right)+\mathcal{J}_{0}\left(-\triangle_{x}+1\right)$. This is a self-adjoint operator in $\mathcal{H}$ with domain $\mathcal{D}(L)=\mathcal{D}\left(L_{0}\right)$. By Lemma 4.3 in Bartsch and Ding [4] and Lemma 2.4 of [16], we have

Lemma 2.3. Let $\left(\mathcal{V}_{0}\right)$ be satisfied. Then one has

(i) $\sigma\left(L_{0}\right)=\sigma_{e}\left(L_{0}\right)$, i.e., $L_{0}$ has only essential spectrum, and $\sigma\left(L_{0}\right) \subset \mathbb{R} \backslash$ $(-1,1)$;

(ii) For every $r \geq 1$, there exist constants $d_{1}, d_{2}>0$ such that

$$
d_{1}\|z\|_{B_{r}}^{r} \leq\left|L_{0} z\right|_{r}^{r}=\int_{{\mathbb{R} \times \mathbb{R}^{N}}^{N}}\left|L_{0} z\right|^{r} \leq d_{2}\|z\|_{B_{r}}^{r} .
$$

for all $z \in B_{r}$. Consequently, $L_{0}: B_{r} \rightarrow L^{r}$ is an isomorphism, $r \geq 1$.

Lemma 2.4. Let $\left(\mathcal{V}_{0}\right)$ be satisfied. Then for all $z \in B_{2}$, we have

$$
c_{1}\left|L_{0} z\right|_{2}^{2} \leq|L z|_{2}^{2} \leq c_{2}\left|L_{0} z\right|_{2}^{2} .
$$

Consequently, for all $z \in B_{2}$

$$
c_{3}\|z\|_{B_{2}}^{2} \leq|L z|_{2}^{2} \leq c_{4}\|z\|_{B_{2}}^{2} .
$$

Proof. Observing that $L=L_{0}+\mathcal{J}_{0}\left(V_{0}-1\right)$, we have

$$
\left|L_{0} z\right|_{2}^{2} \leq 2|L z|_{2}^{2}+2\left|\mathcal{J}_{0}\left(V_{0}-1\right) z\right|_{2}^{2} \leq h|L z|_{2}^{2},
$$

where $h>0$. Thus there is $c_{1}>0$ such that

$$
c_{1}\left|L_{0} z\right|_{2}^{2} \leq|L z|_{2}^{2} .
$$

This yields the left inequality. We now prove the right inequality. Clearly,

$$
|L z|_{2}^{2} \leq 2\left|L_{0} z\right|_{2}^{2}+2\left|\mathcal{J}_{0}\left(V_{0}-1\right) z\right|_{2}^{2} \leq c_{2}\left|L_{0} z\right|_{2}^{2} .
$$

This proves the lemma.

Lemma 2.5. Let $\left(\mathcal{V}_{0}\right)$ and $\left(\mathcal{H}_{0}\right)$ be satisfied. Then for all $z \in B_{2}$, we have

$$
d_{1}|L z|_{2}^{2} \leq\left|L_{\infty} z\right|_{2}^{2} \leq d_{2}|L z|_{2}^{2} .
$$

Consequently, for all $z \in B_{2}$

$$
d_{3}\|z\|_{B_{2}}^{2} \leq\left|L_{\infty} z\right|_{2}^{2} \leq d_{4}\|z\|_{B_{2}}^{2} .
$$


Proof. Since $L_{\infty}=L+B(\infty)$, by Lemma 2.1, it follows that

$$
\begin{aligned}
\left|L_{\infty} z\right|_{2} & =|(L+B(\infty)) z|_{2} \geq|L z|_{2}-|B(\infty) z|_{2} \\
& \geq|L z|_{2}-\frac{\mu_{\infty}}{V_{0}}|L z|_{2}=\left(1-\frac{\mu_{\infty}}{V_{0}}\right)|L z|_{2} .
\end{aligned}
$$

Recall that $\frac{\mu_{\infty}}{V_{0}}<1$. Then there is a positive constant $d_{1}>0$ such that

$$
\left|L_{\infty} z\right|_{2}^{2} \geq d_{1}|L z|_{2}^{2}
$$

On the other hand,

$$
\left|L_{\infty} z\right|_{2}^{2} \leq 2|L z|_{2}^{2}+2|B(\infty) z|_{2}^{2} \leq d_{2}|L z|_{2}^{2} .
$$

Now the conclusion follow.

Remark 2.1. In virtue of Lemmas 2.3-2.5, we write $\mathcal{D}(L)=\mathcal{D}\left(L_{\infty}\right)=B_{2}$ with the graph norm $\|z\|_{L}:=\left|L_{\infty} z\right|_{2}$, which is equivalent to the norm $\|z\|_{B_{2}}$. By the embedding theorem on $B_{2}$ we see that $\mathcal{D}\left(L_{\infty}\right)$ is continuously embedded in $L^{r}\left(\mathbb{R} \times \mathbb{R}^{N}, \mathbb{R}^{2 m}\right)$ for $\mathrm{r}$ satisfying $2 \leq r<\infty$ if $N=1$, and $0 \leq\left(\frac{1}{2}-\frac{1}{r}\right)\left(1+\frac{N}{2}\right) \leq$ 1 if $N \geq 2$. $\mathcal{D}\left(L_{\infty}\right)$ embeds compactly in $L_{l o c}^{r}$ for all $r \geq 2$ if $N=1$, and if $N \geq 2$ for all $r \geq 2$ satisfying $\left(\frac{1}{2}-\frac{1}{r}\right)\left(1+\frac{N}{2}\right)<1$ (see $\left.[5,6]\right)$.

It follows from Lemma 2.2 that $\mathcal{H}$ possesses the orthogonal decomposition

$$
\mathcal{H}=\mathcal{H}^{-} \oplus \mathcal{H}^{+}, \quad z=z^{-}+z^{+}
$$

such that $L_{\infty}$ is negative(resp. positive) definite in $\mathcal{H}^{-}$(resp. $\mathcal{H}^{+}$).

Let $E:=\mathcal{D}\left(\left|L_{\infty}\right|^{\frac{1}{2}}\right)$. Then $\mathrm{E}$ is a Hilbert space under the inner product

$$
\left(z_{1}, z_{2}\right):=\left(\left|L_{\infty}\right|^{\frac{1}{2}} z_{1},\left|L_{\infty}\right|^{\frac{1}{2}} z_{2}\right)_{2},
$$

with the induced norm $\|z\|=(z, z)^{\frac{1}{2}}$. Moreover, E has the following orthogonal decomposition

$$
E=E^{-} \oplus E^{+}, \quad \text { where } E^{ \pm}=E \cap \mathcal{H}^{ \pm}
$$

It is clear that

$$
\left\|z^{ \pm}\right\|^{2} \geq\left(V_{0}-\mu_{\infty}\right)\left|z^{ \pm}\right|_{2}^{2} \quad \text { for all } z \in E .
$$

Let $N^{*}:=\infty$ if $N=1$ and $N^{*}:=\frac{2(N+2)}{N}$ if $N \geq 2$. Then we have

Lemma 2.6. $E$ is continuously embedded in $L^{r}$ for any $r \geq 2$ if $N=1$, and for $r \in\left[2, N^{*}\right]$ if $N \geq 2$. Moreover, $E$ is compactly embedded in $L_{l o c}^{r}$ for all $r \in\left[2, N^{*}\right)$.

Proof. The proof was given in $[4,16]$. We state it here for reader's convenience. We only consider the case $N \geq 2$. It follows by(complex) interpolation that

$$
E=\mathcal{D}\left(\left|L_{\infty}\right|^{\frac{1}{2}}\right) \cong\left[\mathcal{D}\left(L_{\infty}\right), \mathcal{H}\right]_{\frac{1}{2}}
$$

By Remark 2.1, the embeddings

$$
E=\mathcal{D}\left(\left|L_{\infty}\right|^{\frac{1}{2}}\right) \cong\left[\mathcal{D}\left(L_{\infty}\right), \mathcal{H}\right]_{\frac{1}{2}} \hookrightarrow\left[L^{r}, \mathcal{H}\right]_{\frac{1}{2}} \hookrightarrow L^{q}
$$


are continuous for $r=\infty$ if $N=2$, and for $r=\frac{2(N+2)}{N-2}$ if $N \geq 3$, and $\mathrm{q}$ satisfying $\frac{1}{q}=\frac{1}{2}\left(\frac{1}{2}+\frac{1}{r}\right)$, i.e., $q=\frac{2(N+2)}{N}$. For $r \in(2, q)$, the Hölder inequality implies

$$
|z|_{r} \leq|z|_{2}^{1-\beta}|z|_{q}^{\beta} \quad \text { with } \beta=\frac{q(r-2)}{r(q-2)} .
$$

Therefore, E embeds continuously into $L^{r}$ for any for $r \in\left[2, N^{*}\right]$ if $N \geq 2$. Similarly, we see that E is compactly embedded in $L_{l o c}^{r}$ for all $r \in\left[2, N^{*}\right)$.

Set

$$
\Psi(z)=\int_{\mathbb{R} \times \mathbb{R}^{N}} H(t, x, z) .
$$

By assumptions and Lemma 2.6, $\Psi(z) \in C^{1}(E, \mathbb{R})$ and

$$
\Psi^{\prime}(z) v=\int_{\mathbb{R} \times \mathbb{R}^{N}} H_{z}(t, x, z) v, \forall z, v \in E .
$$

Now, let us consider the functional

$$
\Phi(z):=\frac{1}{2}\left\|z^{+}\right\|^{2}-\frac{1}{2}\left\|z^{-}\right\|^{2}-\frac{1}{2} \int_{\mathbb{R} \times \mathbb{R}^{N}} B_{L} z \cdot z-\Psi(z), \quad \forall z=z^{-}+z^{+} \in E,
$$

where $B_{L}$ is defined in $\left(\mathcal{H}_{0}\right)$. Then $\Phi \in C^{1}(E, \mathbb{R})$, and the critical points of $\Phi$ are weak solutions of $(H S)$.

Using the spectrum decomposition of $L_{B}$ one may give $\Phi$ another representation as follows. Note that, by Lemma $2.2, E=\mathcal{D}\left(\left|L_{B}\right|^{\frac{1}{2}}\right)$ is a Hilbert space with the equivalent inner product

$$
(z, v)_{B}:=\left(\left|L_{B}\right|^{\frac{1}{2}} z,\left|L_{B}\right|^{\frac{1}{2}} v\right)_{2}
$$

and norm $\|z\|:=(z, z)_{B}^{\frac{1}{2}}$. Then as above there is a decomposition

$$
E=E_{B}^{-} \oplus E_{B}^{+}
$$

with

$$
\left\|z^{ \pm}\right\|_{B}^{2} \geq\left(V_{0}-\mu_{\infty}-\lambda\right)\left|z^{ \pm}\right|_{2}^{2}, \quad \forall z^{ \pm} \in E_{B}^{ \pm}
$$

Now $\Phi$ can be represented as

$$
\Phi(z)=\frac{1}{2}\left\|z^{+}\right\|_{B}^{2}-\frac{1}{2}\left\|z^{-}\right\|_{B}^{2}-\Psi(z), \quad \forall z=z^{-}+z^{+} \in E_{B}^{-} \oplus E_{B}^{+} .
$$

Now we study the regularity result of the solutions for $(H S)$.

Lemma 2.7. Suppose that the assumptions of Theorem 1.1 hold. Then we have that the critical points of $\Phi$ are element of $B_{r}\left(\mathbb{R} \times \mathbb{R}^{N}, \mathbb{R}^{2 m}\right)$ for all $r \geq 2$.

Proof. If $B(t, x) \equiv 0$, the proof was given in [4,16]. Therefore, in our case, the matrix $B(t, x) \neq 0$ is bounded. The idea of the proof is the same as in Lemma 4.7 of [4], so we outline it for reader's convenience. By [5], we know that the following embedding holds:

$$
B_{q} \hookrightarrow L^{r} \quad \text { is continuous for } q>1, \quad 0 \leq \frac{1}{q}-\frac{1}{r} \leq \frac{2}{N+2} .
$$


Set

$$
\psi(q):= \begin{cases}(N+2) q /(N+2-2 q) & \text { if } 0<q<(N+2) / 2 \\ \infty & \text { if } q \geq(N+2) / 2 .\end{cases}
$$

So $B_{q} \hookrightarrow L^{r}$ is continuous for $1<q \leq r<\psi(q)$ and also for $r=\psi(q)$ if $\psi(q)<\infty$.

Now let $z \in E$ be a weak solution of $(H S)$. Set $w=\mathcal{J}_{0}\left(1-V_{0}\right) z+$ $B(t, x) z+H_{z}(\cdot, \cdot, z)$. Then $z$ is a weak solution of $L_{0} z=w$, hence

$$
z=L_{0}^{-1} w=L_{0}^{-1}\left(\mathcal{J}_{0}\left(1-V_{0}\right) z+B(t, x) z+H_{z}(\cdot, \cdot, z)\right)
$$

Now we define $\chi_{z}: \mathbb{R} \times \mathbb{R}^{N} \rightarrow \mathbb{R}$ by

$$
\chi_{z}(t, x):= \begin{cases}1 & \text { if }|z(t, x)|<1 \\ 0 & \text { if }|z(t, x)| \geq 1\end{cases}
$$

and set

$$
w_{1}(t, x)=\mathcal{J}_{0}\left(1-V_{0}\right) z+B(t, x) z+H_{z}\left(t, x, \chi_{z}(t, x) z(t, x)\right)
$$

and

$$
w_{2}(t, x)=H_{z}\left(t, x,\left(1-\chi_{z}(t, x)\right) z(t, x)\right) .
$$

Then we have $w(t, x)=w_{1}(t, x)+w_{2}(t, x)$. From our assumptions on $B(t, x)$ and $H$, it follows that

$$
\left|w_{1}(t, x)\right| \leq d|z(t, x)|
$$

and

$$
\left|w_{2}(t, x)\right|: \leq \begin{cases}0 & \text { if }|z(t, x)|<1 \\ d|z(t, x)|^{\alpha-1} & \text { if }|z(t, x)| \geq 1,\end{cases}
$$

where $\alpha:=\frac{2 \mu}{\mu-1} \in\left(2, N^{*}\right)$ and $\mu$ is given in $\left(\mathcal{H}_{3}\right)$. Thus $w_{1} \in L^{r}$ for $r \in\left[2, r_{1}\right]$ where $r_{1}:=N^{*}$, and $w_{2} \in L^{r}$ for $r \in\left[1, q_{1}\right]$ where $q_{1}:=\frac{r_{1}}{\alpha-1}$. Here we used that

$$
\operatorname{meas}\left(\left\{(t, x) \in \mathbb{R} \times \mathbb{R}^{N}:|z(t, x)| \geq 1\right\}\right) \leq \int_{\mathbb{R} \times \mathbb{R}^{N}}|z|^{2}<\infty .
$$

Now by Lemma 2.3, we obtain

$$
z_{1}:=L_{0}^{-1} w_{1} \in B_{r} \quad r \in\left[2, r_{1}\right]
$$

and

$$
z_{2}:=L_{0}^{-1} w_{2} \in B_{r} \quad r \in\left[1, q_{1}\right]
$$

We distinguish two cases.

Case 1: $q_{1} \geq N^{*}$. Then $z_{2} \in L^{r}$ for all $r \in\left[q_{1}, \infty\right)$ as a consequence of (2.5). By interpolation we get $z_{2} \in L^{r}$ for $r \geq 2$. Since $r_{1}>q_{1} \geq(N+2) / 2$ we similarly obtain $z_{1} \in L^{r}$ for $r \geq 2$.

Case 2: $q_{1}<N^{*}$. In this case we define inductively $r_{k+1}:=\psi\left(q_{k}\right)$ and $q_{k+1}:=r_{k+1} /(\alpha-1)<r_{k+1}$. Suppose $z_{1} \in B_{r}$ for $r \in\left[2, r_{k}\right]$, and $z_{2} \in B_{r}$ for $r \in\left[2, q_{k}\right]$. Then $z_{1} \in L^{r}$ for $r \in\left[2, \psi\left(r_{k}\right)\right]$, and $z_{2} \in L^{r}$ for $r \in\left[2, \psi\left(q_{k}\right)\right]$. Moreover, since $\psi\left(r_{k}\right)>r_{k+1}$, then $z \in L^{r}$ for $r \in\left[2, r_{k+1}\right]$. This implies 
$w_{1} \in L^{r}$ for $r \in\left[2, r_{k+1}\right]$, and $w_{2} \in L^{r}$ for $r \in\left[2, q_{k+1}\right]$. We claim that there exists $k_{0} \geq 1$ with $q_{k_{0}} \geq(N+2) / 2$. Then we are back in Case 1 and therefore done.

By induction one proves that

$$
r_{k}=\frac{2(N+2)}{N(\alpha-1)^{k-1}-4 \Sigma_{i=1}^{k-2}(\alpha-1)^{k-2}}=\frac{2(N+2)(\alpha-2)}{(\alpha-1)^{k}(N(\alpha-2)-4)+4}
$$

Since $2<\alpha<2(N+2) / N=2+4 / N$ we see that $\psi\left(q_{k-1}\right)<0$ for $k$ large enough. This implies $q_{k-1} \geq(N+2) / 2$ as required.

Lemma 2.8. Assume that $\left(\mathcal{V}_{0}\right)$ and $\left(\mathcal{H}_{0}\right)-\left(\mathcal{H}_{4}\right)$ are satisfied. If $z \in E$ is a solution of $(H S)$ such that

$$
|\Phi(z)| \leq C_{1} \quad \text { and } \quad|z|_{2} \leq C_{2},
$$

then for any $q \geq 2$, we have

$$
z \in B_{q}\left(\mathbb{R} \times \mathbb{R}^{N}, \mathbb{R}^{2 m}\right) \text { and }\|z\|_{B_{q}} \leq \Lambda_{q},
$$

where $\Lambda_{q}$ depends only on $C_{1}, C_{2}$ and $q$.

Proof. The proof is a standard bootstrap argument (see, e.g., [22, Proposition 3.2], or [4], Lemma 4.7 for the iterative steps), so the details are omitted here.

\subsection{The abstract critical point theorem}

In order to study the critical points of $\Phi$, we now recall some generalized linking theorem developed recently in [3]. For related results, see $[6,25]$ and the references therein.

Let $(\mathbb{E},\|\cdot\|)$ be a Banach space with direct sum decomposition $\mathbb{E}=X \oplus Y$ and $P_{X}, P_{Y}$ denote the projections onto $\mathrm{X}, \mathrm{Y}$, respectively. For a functional $\Phi \in C^{1}(\mathbb{E}, \mathbb{R})$, we write $\Phi_{a}:=\{z \in \mathbb{E}: \Phi(z) \geq a\}, \Phi^{b}:=\{z \in \mathbb{E}: \Phi(z) \leq b\}$ and $\Phi_{a}^{b}=\Phi_{a} \cap \Phi^{b}$. Next, let's us recall some definitions:

(i) $\Phi$ is said to be weakly sequentially upper semi-continuous, i.e., $z_{n} \rightarrow z$ in $\mathbb{E}$ one has $\Phi(z) \geq \liminf _{n \rightarrow \infty} \Phi\left(z_{n}\right)$

(ii) $\Phi^{\prime}$ is said to be weakly sequentially continuous, i.e., $\lim _{n \rightarrow \infty} \Phi^{\prime}\left(z_{n}\right) w=$ $\Phi^{\prime}(z) w$ for each $w \in \mathbb{E}$;

(iii) A sequence $\left\{z_{n}\right\} \subset \mathbb{E}$ is said to be a $(C)_{c^{-}}$-sequence if $\Phi\left(z_{n}\right) \rightarrow c$ and $\left(1+\left\|z_{n}\right\|\right) \Phi^{\prime}\left(z_{n}\right) \rightarrow 0$. $\Phi$ is said to satisfy the $(C)_{c^{-}}$condition if any $(C)_{c^{-}}$ sequence has a convergent subsequence.

From now on we assume that the Banach space $\mathrm{X}$ is separable and reflexive, and fix a countable dense subset $B \subset X^{*}$. For each $b \in B$ we define a semi-norm on $\mathbb{E}$ by

$$
P_{b}: \mathbb{E}=X \oplus Y \rightarrow \mathbb{R}, \quad P_{b}(x+y)=q_{b}(x)+\|y\|, \quad \text { for } x+y \in X \oplus Y,
$$

where $q_{b}(x)=\left|(x, b)_{X, X^{*}}\right|=|b(x)|$. We denote by $\mathcal{T}_{B}$ the induced topology. Let $w^{*}$ denote the weak*-topology on $\mathbb{E}^{*}$.

Assume: $z \in \Phi_{c}$.

$\left(\mathcal{A}_{0}\right)$ For any $c \geq 0$, there exists $\xi>0$ such that $\|z\|<\xi\left\|P_{Y} z\right\|$ for all 
$\left(\mathcal{A}_{1}\right)$ For any $c \in \mathbb{R}, \Phi_{c}$ is $\mathcal{T}_{B}$-closed, and $\Phi^{\prime}:\left(\Phi_{c}, \mathcal{T}_{B}\right) \rightarrow\left(\mathbb{E}^{*}, w^{*}\right)$ is continuous.

$\left(\mathcal{A}_{2}\right)$ There exists $\varrho>0$ with $\kappa:=\inf \Phi\left(S_{\varrho} Y\right)>0$ where $S_{\varrho} Y:=\{z \in$ $Y:\|z\|=\varrho\}$.

Then we have the following theorem

Theorem 2.9. Let $\left(\mathcal{A}_{0}\right)-\left(\mathcal{A}_{2}\right)$ be satisfied and suppose there are $R>\varrho>0$ and $e \in Y$ with $\|e\|=1$ such that $\sup \Phi(\partial M) \leq \kappa$ where $M:=\{z=x+$ se; $s \geq$ $0, x \in X,\|z\| \leq R\}$. Then $\Phi$ has a $(C)_{c}$-sequence with $\kappa \leq \Phi(z) \leq \tilde{c}:=$ $\sup \Phi(M)$. Moreover, if $\Phi$ satisfies the $(C)_{c}$-condition for all $c \leq \tilde{c}$, then $\Phi$ has a critical point $z$ with $\kappa \leq \Phi(z) \leq \tilde{c}$.

This theorem is a special case of the theorem 4.4 of [3]. In the following, we will use Theorem 2.9 to prove our Theorem 1.1. The key is to verify the above conditions $\left(\mathcal{A}_{0}\right)-\left(\mathcal{A}_{2}\right)$, and the following lemma is useful to verify $\left(\mathcal{A}_{1}\right)$ (see [18] or [3]).

Lemma 2.10. Let

$$
\Phi(u)=\frac{1}{2}\|y\|^{2}-\frac{1}{2}\|x\|^{2}-\Psi(u), \text { for } u=x+y \in \mathbb{E}=X \oplus Y .
$$

Suppose

(1) $\Psi \in C^{1}(\mathbb{E}, \mathbb{R})$ is bounded from below;

(2) $\Psi:\left(\mathbb{E}, \mathcal{T}_{w}\right) \rightarrow \mathbb{R}$ is sequentially lower semicontinuous, that is, $u_{n} \rightarrow u$ in $\mathbb{E}$ implies $\Psi(u) \leq \liminf _{n \rightarrow \infty} \Psi\left(u_{n}\right) ;$

(3) $\Psi^{\prime}:\left(\mathbb{E}, \mathcal{T}_{w}\right) \rightarrow\left(\mathbb{E}, \mathcal{T}_{w^{*}}\right)$ is sequentially continuous;

(4) $\nu: \mathbb{E}: \rightarrow \mathbb{R}, \nu(u):=\|u\|^{2}$ is $C^{1}$ and $\nu^{\prime}:\left(\mathbb{E}, \mathcal{T}_{w}\right) \rightarrow\left(\mathbb{E}, \mathcal{T}_{w^{*}}\right)$ is sequentially continuous.

Then $\Phi$ satisfies $\left(\mathcal{A}_{1}\right)$.

\section{The limit equation}

In this section we study the following autonomous equation

$$
\mathcal{J} \frac{d}{d t} z+\mathcal{J}_{0} \mathcal{S} z+\mathcal{J} b \cdot z+B(\infty) z=H_{\infty}(z)
$$

where $H_{\infty}$ is given in $\left(\mathcal{H}_{4}\right)$.

In the following for notational simplicity, we write $\hat{x}=(t, x)$. For $0 \neq k=$ $\left(k_{0}, k_{1}, \ldots, k_{N}\right) \in \mathbb{Z}^{N+1}$, define $k * z(t, x):=z\left(t+k_{0}, x_{1}+k_{1}, \ldots, x_{N}+k_{N}\right)$. By the virtue of $\left(\mathcal{H}_{1}\right)-\left(\mathcal{H}_{4}\right)$, we first have the following lemma.

Lemma 3.1. The limit $H_{\infty}$ possesses the following properties.

(i) $H_{\infty}(0)=0, H_{\infty}(z)>0$ if $z \neq 0$, and $H_{\infty}^{\prime}(z)=o(z)$ as $|z| \rightarrow 0$.

(ii) $H_{\infty}(z)|z|^{-2} \rightarrow \infty$ as $|z| \rightarrow \infty$.

(iii) $\tilde{H}_{\infty}(z)>0$ if $z \neq 0$, and $\left|H_{\infty}^{\prime}(z)\right|^{\mu} \leq c_{1} \tilde{H}_{\infty}(z)|z|^{\mu}$ if $|z| \geq r_{1}$, where $\mu$ is defined in $\left(\mathcal{H}_{3}\right)$.

(iv) $H_{\infty}(z)$ is strictly convex in $\mathbb{R}^{2 m} \backslash\{0\}$. 
Proof. Both $H_{\infty}(0)=0$ and $H_{\infty}^{\prime}(z)=o(z)$ as $|z| \rightarrow 0$ are assumed in $\left(\mathcal{H}_{4}\right)$. By $\left(\mathcal{H}_{3}\right)$ one has $\tilde{H}(t, x, z) \geq 0$, which together with $\left(\mathcal{H}_{1}\right)$ implies that $\frac{1}{2} H_{z}(t, x, z) z \geq H(t, x, z) \geq 0$, hence $H(t, x, 0)=0$. Since $\tilde{H}(t, x, z)>0$ if $z \neq 0$, we see that $H(t, x, z)=\int_{0}^{1} H_{z}(t, x, \tau z) z d \tau>0$ if $z \neq 0$. Observe that

$$
0 \leq H(t, x, z)-H_{\infty}(z)=\int_{0}^{1}\left(H_{z}(t, x, \tau z)-H_{\infty}^{\prime}(\tau z)\right) z d \tau .
$$

It follows from $\left(\mathcal{H}_{4}\right)$ that $H(t, x, z) \rightarrow H_{\infty}(z)$ uniformly in bounded sets of $z$ as $|\hat{x}| \rightarrow \infty$. This, jointly with that $\inf _{(t, x) \in \mathbb{R} \times \mathbb{R}^{N}} \tilde{H}(t, x, z)>0$ if $z \neq 0$, implies

$$
\tilde{H}_{\infty}(z)>0 \text { if } z \neq 0
$$

Hence $H_{\infty}^{\prime}(z) z>0$ and

$$
H_{\infty}(z)=\int_{0}^{1} H_{\infty}^{\prime}(\tau z) z d \tau>0 \quad \text { if } z \neq 0
$$

which shows $(i)$.

By $\left(\mathcal{H}_{2}\right)$, for any $K>0$ there is $b_{K}>0$ such that $H(t, x, z)|z|^{-2} \geq K$ if $|z| \geq b_{K}$. Observe that

$$
\begin{aligned}
\frac{H_{\infty}(z)}{|z|^{2}} & =\frac{H_{\infty}(z)-H(t, x, z)}{|z|^{2}}+\frac{H(t, x, z)}{|z|^{2}} \\
& \geq \frac{H_{\infty}(z)-H(t, x, z)}{|z|^{2}}+K
\end{aligned}
$$

for all $|z| \geq b_{K}$. Letting $|\hat{x}| \rightarrow \infty$, we get that $H_{\infty}(z)|z|^{-2} \geq K$ for all $|z| \geq b_{K}$, proving $(i i)$.

Note that by $\left(\mathcal{H}_{3}\right)$ for all $|z| \geq r_{1}$, we have

$$
\begin{aligned}
\frac{\left|H_{\infty}^{\prime}(z)\right|}{|z|} & \leq \frac{\left|H_{\infty}^{\prime}(z)-H_{z}(t, x, z)\right|}{|z|}+\frac{\left|H_{z}(t, x, z)\right|}{|z|} \\
& \leq \frac{\left|H_{\infty}^{\prime}(z)-H_{z}(t, x, z)\right|}{|z|}+c_{1}^{\frac{1}{\mu}}(\tilde{H}(t, x, z))^{\frac{1}{\mu}}
\end{aligned}
$$

Fixed arbitrarily $|z| \geq r_{1}$, letting $|\hat{x}| \rightarrow \infty$ yields

$$
\frac{\left|H_{\infty}^{\prime}(z)\right|}{|z|} \leq c_{1}^{\frac{1}{\mu}} \tilde{H}_{\infty}(z)^{\frac{1}{\mu}}
$$

This together with (3.2) implies the conclusion (iii).

Finally, by (3.2) again, $H_{\infty}^{\prime}(z) z>0$ if $z \neq 0$. Using the conclusion $(i i)$ of $\left(\mathcal{H}_{4}\right)$ with $w \neq 0, y=0$ one gets $H_{\infty}^{\prime \prime}(w) w \cdot w \geq H_{\infty}^{\prime}(w) w>0$, this proves (iv).

As a consequence one sees easily that, for any $\varepsilon>0$, there is $C_{\varepsilon}>0$ satisfying

$$
\frac{\left|H_{\infty}^{\prime}(z)\right|}{|z|} \leq \varepsilon+C_{\varepsilon} \tilde{H}_{\infty}(z)^{\frac{1}{\mu}}, \quad \text { for all } z \neq 0
$$


In the following, we consider $\frac{2 \mu}{\mu-1} \leq p<N^{*}$. Note that by the assumption $\left(\mathcal{H}_{3}\right)$, one has $2<\frac{2 \mu}{\mu-1}<N^{*}$ if $N \geq 2$, and

$$
\left|H_{\infty}^{\prime}(z)\right| \leq \varepsilon|z|+C_{\varepsilon}|z|^{p-1} \quad \text { and } \quad\left|H_{\infty}(z)\right| \leq \varepsilon|z|^{2}+C_{\varepsilon}|z|^{p}
$$

for all $z \in E$.

Set

$$
\Psi_{\infty}(z)=\int_{\mathbb{R} \times \mathbb{R}^{N}} H_{\infty}(z)
$$

and define the functional

$$
F(z):=\frac{1}{2}\left\|z^{+}\right\|^{2}-\frac{1}{2}\left\|z^{-}\right\|^{2}-\Psi_{\infty}(z), \quad \forall z=z^{-}+z^{+} \in E .
$$

It follows from the assumptions on $H_{\infty}(z)$ that $F \in C^{1}(E, \mathbb{R})$ and its critical points are solutions of (3.1). Moreover, by Lemmas 2.6 and 3.1, we have the following results.

Lemma 3.2. Under the assumptions of Theorem 1.1, one has

(1) $\Psi_{\infty}$ is weakly sequentially lower semicontinuous and $F^{\prime}$ is weakly sequentially continuous;

(2) For any finite dimensional subspace $Z \subset E^{+}$,

$$
F(z) \rightarrow-\infty \quad \text { as } z \in E^{-} \oplus Z, \quad\|z\| \rightarrow \infty
$$

(3) There are $\rho>0$ and $\kappa>0$ such that

$$
\left.F(z)\right|_{K_{\rho} \cap E^{+}} \geq 0 \quad \text { and }\left.\quad F(z)\right|_{\partial K_{\rho} \cap E^{+}} \geq \kappa,
$$

where $K_{\rho}:=\{z \in E ;\|z\| \leq \rho\}$.

Proof. The proof was given in $[16,18]$, here we omit the details.

Lemma 3.3. Let $\left\{z_{j}\right\}$ be a $(C)_{c}$-sequence for $F$. Then it is bounded and $c \geq 0$. Moreover, there is a subsequence, denoted again by $\left\{z_{j}\right\}$, satisfying $z_{j} \rightarrow z$, $F\left(z_{j}-z\right) \rightarrow c-F(z)$ and $F^{\prime}\left(z_{j}-z\right) \rightarrow 0$.

Proof. First, by Lemma 3.1, a standard argument shows that $\left\{z_{j}\right\}$ is bounded (see, e.g., [18]). Next, by the conclusion (iii) of Lemma 3.1, it holds that

$$
F\left(z_{j}\right)-\frac{1}{2} F^{\prime}\left(z_{j}\right) z_{j}=\int_{\mathbb{R} \times \mathbb{R}^{N}} \tilde{H}_{\infty}\left(z_{j}\right) \geq 0 .
$$

Taking the limit yields $c \geq 0$.

Finally, by Lemma 5.4 of [16], we know that $F\left(z_{j}-z\right) \rightarrow c-F(z)$ and $F^{\prime}\left(z_{j}-z\right) \rightarrow 0$.

Let $\mathcal{K}:=\left\{z \in E: F^{\prime}(z)=0\right\}$ be the critical set of $F$.

Lemma 3.4. $\mathcal{K} \backslash\{0\} \neq 0, \mathcal{K} \subset \cap_{r \geq 2} B_{r}$ and

$$
\hat{C}:=\inf \{F(z): z \in \mathcal{K} \backslash\{0\}\}>0
$$


Proof. In the following, we will use Theorem 2.9 to prove that $\mathcal{K} \backslash\{0\} \neq \emptyset$. First we verify that the functional $F$ satisfies the conditions $\left(\mathcal{A}_{0}\right)-\left(\mathcal{A}_{2}\right)$. Let $X=E^{-}$and $Y=E^{+}$, one has $E=X \oplus Y$. Let $F_{0}=\{z \in E: F(z) \geq 0\}$. Since $H_{\infty}(z) \geq 0$, for $z \in F_{0}$ one has

$$
0 \leq \frac{1}{2}\left\|z^{+}\right\|^{2}-\frac{1}{2}\left\|z^{-}\right\|^{2}-\int_{\mathbb{R}^{N}} H_{\infty}(z) \leq \frac{1}{2}\left\|z^{+}\right\|^{2}-\frac{1}{2}\left\|z^{-}\right\|^{2},
$$

which implies $\left\|z^{-}\right\|^{2} \leq\left\|z^{+}\right\|^{2}$. Then there exists $\xi>\sqrt{2}$ such that $\|z\| \leq$ $\xi\left\|z^{+}\right\|$, and $F$ satisfies $\left(\mathcal{A}_{0}\right)$. By virtue of Lemma 2.10 and the conclusion (1) of Lemma 3.2, it follows that $\left(\mathcal{A}_{1}\right)$ holds. From the conclusion (3) of Lemma 3.2, we know that $\left(\mathcal{A}_{2}\right)$ holds. Let $e \in E^{+}$with $\|e\|=1$. By the conclusion (2) of Lemma 3.2, we have that there exist $R>\rho>0$ such that $\left.F\right|_{\partial M} \leq 0$, where

$$
M=\left\{z=z^{-}+s e: z^{-} \in E^{-}, s \geq 0,\|z\| \leq R\right\} .
$$

Thus all of the conditions of Theorem 2.9 hold. Then we have that there is

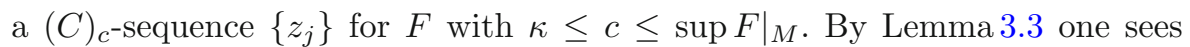
that $\left\{z_{j}\right\}$ is bounded in E. By the concentration principle [26], a standard argument shows that $\left\{z_{j}\right\}$ is non-vanishing, that is, there exist $r, \eta>0$ and $\left\{a_{j}\right\} \subset \mathbb{Z}^{1+N}$ such that $\lim \sup _{j \rightarrow \infty} \int_{D\left(a_{j}, r\right)}\left|z_{j}\right|^{2} \geq \eta$, where $D\left(a_{j}, r\right):=\{\hat{x} \in$ $\left.\mathbb{R} \times \mathbb{R}^{N}:\left|\hat{x}-a_{j}\right| \leq r\right\}$. Set $v_{j}=z_{j} * a_{j}$. It follows the invariance of the norm and the functional under the $*$-action that $\left\|v_{j}\right\|=\left\|z_{j}\right\| \leq C$ and $F\left(v_{j}\right) \rightarrow c \geq \kappa$, $F^{\prime}\left(v_{j}\right) \rightarrow 0$. Therefore, $v_{j} \rightarrow v$ with $v \neq 0$ and $F^{\prime}(v)=0$, that is, $v$ is a nontrivial solution of (3.1). Thus,

$$
\mathcal{K} \backslash\{0\} \neq 0 .
$$

As in Lemma 2.7 one sees that $\mathcal{K} \subset B_{r}$ for $r \geq 2$.

If $z \in \mathcal{K}$, one has

$$
F(z)-\frac{1}{2} F^{\prime}(z) z=\int_{\mathbb{R} \times \mathbb{R}^{N}} \tilde{H}_{\infty}(z) \geq 0 .
$$

For proving $\hat{C}>0$, assume by contradiction that $\hat{C}=0$. Let $z_{j} \in \mathcal{K} \backslash\{0\}$ be such that $F\left(z_{j}\right) \rightarrow 0$. Then $\left\{z_{j}\right\}$ is a $(C)_{0}$-sequence, hence it is bounded by Lemma 3.3. We can assume that $z_{j} \rightarrow z \in \mathcal{K}$. Then

$$
F\left(z_{j}\right)=\int_{\mathbb{R} \times \mathbb{R}^{N}} \tilde{H}_{\infty}\left(z_{j}\right) \rightarrow 0 .
$$

By (3.3) and Hölder inequality $\left(\frac{1}{\mu}+\frac{1}{\mu^{\prime}}=1, \mu=\frac{p}{p-2}\right)$, one sees that for any $\varepsilon>0$

$$
\begin{aligned}
\left\|z_{j}\right\|^{2} & =\int_{\mathbb{R} \times \mathbb{R}^{N}} H_{\infty}^{\prime}\left(z_{j}\right)\left(z_{j}^{+}-z_{j}^{-}\right) \\
& \leq \varepsilon\left|z_{j}\right|_{2}^{2}+C_{\varepsilon} \int_{{\mathbb{R} \times \mathbb{R}^{N}}_{\infty}} \tilde{H}_{\infty}\left(z_{j}\right)^{\frac{1}{\mu}}\left|z_{j}\right|\left|z_{j}^{+}-z_{j}^{-}\right| \\
& \leq \varepsilon\left|z_{j}\right|_{2}^{2}+C_{\varepsilon}\left(\int_{\mathbb{R} \times \mathbb{R}^{N}} \tilde{H}_{\infty}\left(z_{j}\right)\right)^{\frac{1}{\mu}}\left|z_{j}\right|_{p}\left(\int_{\mathbb{R} \times \mathbb{R}^{N}}\left|z_{j}^{+}-z_{j}^{-}\right|^{p}\right)^{\frac{1}{p}} \\
& \leq c \varepsilon\left\|z_{j}\right\|^{2}+c C_{\varepsilon} F\left(z_{j}\right)^{\frac{1}{\mu}}\left\|z_{j}\right\|^{2} .
\end{aligned}
$$


Hence $1 \leq c \varepsilon+o(1)$, a contradiction.

Finally, we show that there is $\left\{z_{j}\right\} \in \mathcal{K} \backslash\{0\}$ with $F\left(z_{j}\right) \rightarrow \hat{C}$. Then, as before, $\left\{z_{j}\right\}$ is bounded, and by applying the concentration principle one may assume $z_{j} \rightarrow z \in \mathcal{K} \backslash\{0\}$. Now

$$
\hat{C}=\lim _{j \rightarrow \infty} F\left(z_{j}\right)=\lim _{j \rightarrow} \int_{\mathbb{R}_{\mathbb{R}^{N}}} \tilde{H}_{\infty}\left(z_{j}\right) \geq \int_{\mathbb{R} \times \mathbb{R}^{N}} \tilde{H}_{\infty}(z)=F(z) \geq \hat{C},
$$

that is, $F(z)=\hat{C}$.

In the following, we follow the idea of $[14,15]$ to establish the linking levels for the functional $\Phi(z)$. Following Ackermann [1], for fixed $u \in E^{+}$, we introduce the functional $\psi_{u}: E^{-} \rightarrow \mathbb{R}$ by

$$
\psi_{u}(v):=F(u+v)=\frac{1}{2}\|u\|^{2}-\frac{1}{2}\|v\|^{2}-\Psi_{\infty}(u+v) .
$$

One has

$$
\begin{aligned}
\psi_{u}^{\prime \prime}(v)[w, w] & =-\|w\|^{2}-\Psi_{\infty}^{\prime \prime}(u+v)[w, w] \\
& =-\|w\|^{2}-\int_{\mathbb{R} \times \mathbb{R}^{N}} H_{\infty}^{\prime \prime}(u+v) w \cdot w
\end{aligned}
$$

for all $v, w \in E^{-}$, which implies that $\psi_{u}(\cdot)$ is strictly concave. Moreover,

$$
\psi_{u}(v) \leq \frac{1}{2}\left(\|u\|^{2}-\|v\|^{2}\right) \rightarrow-\infty, \quad \text { as }\|v\| \rightarrow \infty .
$$

Clearly, it follows from Lemma 3.2 that $\psi_{u}$ is weak sequential upper semicontinuous. Thus there is a unique strict maximum point $f(u)$ for $\psi_{u}(\cdot)$ which is also the only critical point of $\psi_{u}$ on $E^{-}$and satisfies:

$$
\begin{aligned}
& v \neq f(u) \Leftrightarrow F(u+v)<F(u+f(u)), \\
& v=f(u) \Leftrightarrow \psi_{u}^{\prime}(f(u)) w=0, \text { i.e. }, \\
& \quad(f(u), w)+\int_{\mathbb{R} \times \mathbb{R}^{N}} H_{\infty}^{\prime}(u+f(u)) w=0
\end{aligned}
$$

for all $u \in E^{+}$and $v, w \in E^{-}$.

By Lemma 5.6 of [1], we have the following results.

Lemma 3.5. Under the conditions of Theorem 1.1, we have:

(i) $f$ is $\mathbb{R} \times \mathbb{R}^{N}$-invariant, i.e., $f(a * u)=f(u)$ for all $a \in \mathbb{R} \times \mathbb{R}^{N}$;

(ii) $f \in C^{1}\left(E^{+}, E^{-}\right)$and $f(0)=0$;

(iii) $f$ is a bounded map;

(iv) If $u_{n} \rightarrow u$, then $f\left(u_{n}\right)-f\left(u_{n}-u\right) \rightarrow f(u)$ and $f\left(u_{n}\right) \rightarrow f(u)$. The same is true for $|f(u)|_{2}^{2}$. 
Now we define the reduce functional $I(u):=F(u+f(u))=\frac{1}{2}\|u\|^{2}-$ $\frac{1}{2}\|f(u)\|^{2}-\Psi_{\infty}(u+f(u))$. By (3.6), we have

$$
\begin{aligned}
I^{\prime}(u) v & =(u, v)-\left(f(u), f^{\prime}(u) v\right)-\int_{\mathbb{R}_{\mathbb{R}^{N}}} H_{\infty}^{\prime}(u+f(u))\left(v+f^{\prime}(u) v\right) \\
& =F^{\prime}(u+f(u))(v+f(v)) \\
& =(u, v)-(u, f(v))-\int_{\mathbb{R}^{\prime} \mathbb{R}^{N}} H_{\infty}^{\prime}(u+f(u))(v+f(v))
\end{aligned}
$$

for all $u, v \in E^{+}$. Then we know that the critical points of $I$ and $F$ are in one to one correspondence via the injective map $u \rightarrow u+f(u)$ from $E^{+}$into $E^{-}$. That is, letting

$$
\mathcal{K}^{+}:=\left\{u \in E^{+}: I^{\prime}(u)=0\right\}
$$

one has

$$
\mathcal{K}:=\left\{u+f(u): u \in \mathcal{K}^{+}\right\}
$$

In particular,

$$
\hat{C}=\inf \left\{I(u): u \in \mathcal{K}^{+} \backslash\{0\}\right\} .
$$

Lemma 3.6. If $\left\{u_{j}\right\} \subset E^{+}$is a $(C)_{c}$-sequence for $I$, then $z_{j}:=u_{j}+f\left(u_{j}\right) \in E$

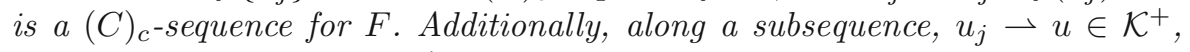
$I\left(u_{j}-u\right) \rightarrow c-I(u)$ and $I^{\prime}\left(u_{j}-u\right) \rightarrow 0$.

Proof. It is clear by definition that

$$
F\left(z_{j}\right)=F\left(u_{j}+f\left(u_{j}\right)\right)=I\left(u_{j}\right) \rightarrow c,
$$

by (3.6), for any $w=w^{-}+w^{+} \in E$ one has

$$
\begin{aligned}
F^{\prime}\left(z_{j}\right) w & =\left(u_{j}, w^{+}\right)-\left(f\left(u_{j}\right), w^{-}\right)-\int_{\mathbb{R}_{\mathbb{R}^{N}}} H_{\infty}^{\prime}\left(z_{j}\right)\left(w^{-}\right)-\int_{\mathbb{R} \times \mathbb{R}^{N}} H_{\infty}^{\prime}\left(z_{j}\right)\left(w^{+}\right) \\
& =\left(u_{j}, w^{+}\right)-\int_{\mathbb{R} \times \mathbb{R}^{N}} H_{\infty}^{\prime}\left(z_{j}\right)\left(w^{+}\right) \\
& =I^{\prime}\left(u_{j}\right) w^{+} .
\end{aligned}
$$

Thus $\left(1+\left\|z_{j}\right\|\right) F^{\prime}\left(z_{j}\right) \rightarrow 0$. The last conclusion follows from Lemmas 3.3 and 3.5 .

In the next, we discuss the mountain pass geometry of functional $I$. Obviously, one has the following

Lemma 3.7. I possesses the mountain pass geometry:

(1) There is $\rho>0$ such that inf $I\left(E^{+} \cap \partial K_{\rho}\right)>0$, where $\partial K_{\rho}=\{z \in E$ : $\|z\|=\rho\}$

(2) For any finite dimensional subspace $X \subset E^{+}, I(u) \rightarrow-\infty$ as $u \in X$, $\|u\| \rightarrow \infty$. 
Proof. (1) By (3.4), for $u \in E^{+}$one has

$$
\begin{aligned}
I(u) & =\frac{1}{2}\|u\|^{2}-\frac{1}{2}\|f(u)\|^{2}-\int_{\mathbb{R} \times \mathbb{R}^{N}} H_{\infty}(u+f(u)) \\
& =\frac{1}{2}\|u\|^{2}+(F(u+f(u))-F(u))-\int_{\mathbb{R}^{\prime} \mathbb{R}^{N}} H_{\infty}(u) \\
& \geq \frac{1}{2}\|u\|^{2}-\int_{\mathbb{R} \times \mathbb{R}^{N}} H_{\infty}(u) \\
& \geq \frac{1}{2}\|u\|^{2}-\varepsilon|u|_{2}^{2}-C_{\varepsilon}|u|_{p}^{p}
\end{aligned}
$$

and the result follows from Lemma 2.6.

(2) Arguing indirectly, assume that for some $u_{j} \subset X$ with $\left\|u_{j}\right\| \rightarrow \infty$. There is $K>0$ such that $I\left(u_{j}\right) \geq-K$ for all $j$. Let $v_{j}:=u_{j}+f\left(u_{j}\right)$, then it follows from $\left\|u_{j}\right\| \leq\left\|v_{j}\right\|$ that $\left\|v_{j}\right\| \rightarrow \infty$ as $j \rightarrow \infty$. Setting $w_{j}:=\frac{v_{j}}{\left\|v_{j}\right\|}$, by Lemma 3.5, we have that $\left\|w_{j}\right\|=1, w_{j} \rightarrow w, w_{j}^{-} \rightarrow w^{-}, w_{j}^{+} \rightarrow w^{+}$ and

$$
-\frac{K}{\left\|v_{j}\right\|^{2}} \leq \frac{I\left(u_{j}\right)}{\left\|v_{j}\right\|^{2}}=\frac{F\left(v_{j}\right)}{\left\|v_{j}\right\|^{2}}=\frac{1}{2}\left\|w_{j}^{+}\right\|^{2}-\frac{1}{2}\left\|w_{j}^{-}\right\|^{2}-\int_{\mathbb{R} \times \mathbb{R}^{N}} \frac{H_{\infty}\left(v_{j}\right)}{\left\|v_{j}\right\|^{2}} .
$$

We claim that $w^{+} \neq 0$. Indeed, if not then it follows from (3.8) that

$$
0 \leq \frac{1}{2}\left\|w_{j}^{-}\right\|^{2}+\int_{\mathbb{R} \times \mathbb{R}^{N}} \frac{H_{\infty}\left(v_{j}\right)}{\left\|v_{j}\right\|^{2}} \leq \frac{1}{2}\left\|w_{j}^{+}\right\|^{2}+\frac{K}{\left\|v_{j}\right\|^{2}} \rightarrow 0 .
$$

This implies that $\left\|w_{j}^{-}\right\| \rightarrow 0$, hence $1=\left\|w_{j}\right\|^{2} \rightarrow 0$, a contradiction.

Since $X$ is a finite subspace of $E^{+}$, then the exists a best constant $\omega>0$ such that $\left\|w^{+}\right\|^{2} \leq \omega\left|w^{+}\right|_{2}^{2}$. By the conclusion (ii) of Lemma 3.1, then there is $R>0$ such that $H_{\infty}(z) \geq 2 \omega|z|^{2}$ if $|z| \geq R$. Observing that

$$
\left\|w^{+}\right\|^{2}-\left\|w^{-}\right\|^{2}-2 \omega|w|_{2}^{2} \leq-\left(\omega\left|w^{+}\right|_{2}^{2}+\left\|w^{-}\right\|^{2}+2 \omega\left|w^{-}\right|_{2}^{2}\right)<0
$$

hence, there is a bounded set $D \subset \mathbb{R} \times \mathbb{R}^{N}$ such that

$$
\left\|w^{+}\right\|^{2}-\left\|w^{-}\right\|^{2}-2 \omega \int_{D}|w|_{2}<0 .
$$

Then

$$
\begin{aligned}
\frac{I\left(u_{j}\right)}{\left\|v_{j}\right\|^{2}} & \leq \frac{1}{2}\left(\left\|w_{j}^{+}\right\|^{2}-\left\|w_{j}^{-}\right\|^{2}\right)-\int_{D} \frac{H_{\infty}\left(v_{j}\right)}{\left\|v_{j}\right\|^{2}} \\
& =\frac{1}{2}\left(\left\|w_{j}^{+}\right\|^{2}-\left\|w_{j}^{-}\right\|^{2}-2 \omega \int_{D}\left|w_{j}\right|_{2}\right)-\int_{D} \frac{H_{\infty}\left(v_{j}\right)-\omega\left|v_{j}\right|^{2}}{\left\|v_{j}\right\|^{2}} \\
& \leq \frac{1}{2}\left(\left\|w_{j}^{+}\right\|^{2}-\left\|w_{j}^{-}\right\|^{2}-2 \omega \int_{D}\left|w_{j}\right|_{2}\right)+\frac{\omega R^{2}|D|}{\left\|v_{j}\right\|^{2}}
\end{aligned}
$$

Hence, letting $j \rightarrow \infty$ yields

$$
0 \leq\left\|w^{+}\right\|^{2}-\left\|w^{-}\right\|^{2}-2 \omega \int_{D}|w|_{2}<0
$$

which is contradiction. 
Lemma 3.4 implies 0 is an isolated critical point of $I$. Therefore there is $k>0$ such that $\|w\| \geq k$ for all nontrivial critical points $w$ of $I$. Below we set

$$
\mathcal{N}^{+}:=\left\{u \in E^{+} \backslash\{0\}: I^{\prime}(u) u=0\right\} .
$$

Lemma 3.8. For each $u \in E^{+} \backslash\{0\}$, there is a unique $s=s(u)>0$ such that $s u \in \mathcal{N}^{+}$.

Proof. We follow the idea of the proof [1]. Observe that,

$$
\nabla I(u)=\nabla F(u+f(u))=\mathcal{P}^{+} \nabla F(u+f(u))
$$

and

$$
\nabla^{2} I(u)=\nabla^{2} F(u+f(u))\left(I+f^{\prime}(u)\right)=\mathcal{P}^{+} \nabla F(u+f(u))\left(I+f^{\prime}(u)\right)
$$

where $\mathcal{P}^{ \pm}: E \rightarrow E^{ \pm}$denote the orthogonal projector. Fixed $z \in E^{+} \backslash\{0\}$ with $I^{\prime}(z) z=0$, and set $u=z+f(z)$ and $v=f^{\prime}(z) z-f(z) \in E^{-}$. Then $u \neq 0$, by (3.6) and $\left(\mathcal{H}_{4}\right)$, we get

$$
\begin{aligned}
I^{\prime \prime}(z)[z, z]= & \left(\nabla^{2} I(z) z, z\right) \\
= & \left(\nabla^{2} F(u)\left[z+f^{\prime}(z) z\right], z+f^{\prime}(z) z\right) \\
= & \left(\nabla^{2} F(u)[u+v], u+v\right) \\
= & \left(\nabla^{2} F(u) u, u\right)+2\left(\nabla^{2} F(u) u, v\right)+\left(\nabla^{2} F(u) v, v\right) \\
= & \left\|u^{+}\right\|^{2}-\left\|u^{-}\right\|^{2}-\left(\nabla^{2} \Psi_{\infty}(u) u, u\right) \\
& +2\left(u^{+}-u^{-}-\nabla^{2} \Psi_{\infty}(u) u, v\right)+\left(-v-\nabla^{2} \Psi_{\infty}(u) v, v\right) \\
= & \left(\nabla \Psi_{\infty}(u)-\nabla^{2} \Psi_{\infty}(u) u, u\right)+2\left(\nabla \Psi_{\infty}(u)-\nabla^{2} \Psi_{\infty}(u) u, v\right) \\
& -\left(\nabla \Psi_{\infty}(u) v, v\right)-\|v\|^{2} \\
= & \left(\Psi_{\infty}^{\prime}(u) u-\Psi_{\infty}^{\prime \prime}(u)[u, u]\right)+2\left(\Psi_{\infty}^{\prime}(u) v-\Psi_{\infty}^{\prime \prime}(u)[u, v]\right) \\
= & -\Psi_{\infty}^{\prime \prime}(u)[v, v]-\|v\|^{2} \\
= & -\int_{\mathbb{R} \times \mathbb{R}^{N}}\left(H_{\infty}^{\prime \prime}(u)(u+v)(u+v)-H_{\infty}^{\prime}(u)(u+2 v)\right)-\|v\|^{2}<0 .
\end{aligned}
$$

Now let $u \in E^{+} \backslash\{0\}$ and $h(s)=I(s u)$, one has $h(0)=0, h(s)>0$ for $s>0$ sufficiently small, and by Lemma 3.7, we know that $h(s) \rightarrow-\infty$ as $s \rightarrow \infty$. Thus there is $s(u)>0$ such that

$$
I(s(u) u)=\sup _{s \geq 0} I(s u) .
$$

Clearly, we have

$$
\left.\frac{d I(s u)}{d s}\right|_{s=s(u)}=I^{\prime}(s(u) u) u=\frac{1}{s(u)} I^{\prime}(s(u) u) s u=0,
$$

and consequently by (3.9)

$$
I^{\prime \prime}(s(u) u)(s(u) u)<0 .
$$

One sees that $\operatorname{such} s(u)>0$ is unique. 
Set

$$
\begin{aligned}
& b_{1}:=\inf \left\{I(u): u \in \mathcal{N}^{+}\right\}, \\
& b_{2}:=\inf _{\gamma \in \mathcal{M}} \sup _{s \in[0.1]} I(\gamma(s))
\end{aligned}
$$

where

$$
\mathcal{M}:=\left\{\gamma \in C\left([0,1], E^{+}\right): \gamma(0)=0, I(\gamma(1))<0\right\} .
$$

Lemma 3.9. $\hat{C}=b_{1}=b_{2}$.

Proof. In the following, we shall show that $b_{1} \leq \hat{C} \leq b_{2} \leq b_{1}$.

$b_{1} \leq \hat{C}$. This holds because $\mathcal{K}^{+} \backslash\{0\} \subset \mathcal{N}^{+}$and (3.7) holds.

$\hat{C} \leq b_{2}$. Let $\left\{u_{j}\right\} \subset E^{+}$be a mountain pass sequence, that is, $I\left(u_{j}\right) \rightarrow b_{2}$ and $\left(1+\left\|u_{j}\right\|\right) I^{\prime}\left(u_{j}\right) \rightarrow 0$. It is not difficult to check that $\left\{u_{j}\right\}$ is bounded in E. By the concentration compactness principle, a standard argument shows that $\left\{u_{j}\right\}$ is non-vanishing, that is, there exist $k, \eta>0$ and $\left\{a_{j}\right\} \subset \mathbb{Z}^{N+1}$

$$
\limsup _{j \rightarrow \infty} \int_{D\left(a_{j}, r\right)}\left|u_{j}\right|^{2} \geq \eta,
$$

where $D\left(a_{j}, r\right)=\left\{\hat{x} \in \mathbb{R} \times \mathbb{R}^{N}:\left|\hat{x}-a_{j}\right| \leq r\right\}$. Set $v_{j}:=a_{j} * u_{j}$. It follows from the invariance of the norm and of the functional under the $*$-action that $I\left(v_{j}\right) \rightarrow b_{2}$ and $\left(1+\left\|v_{j}\right\|\right) I^{\prime}\left(v_{j}\right) \rightarrow 0$. Then it follows from Lemma 3.6 that, along a subsequence, $v_{j} \rightarrow v \in \mathcal{K}^{+} \backslash\{0\}$ with $I\left(v_{j}-v\right) \rightarrow b_{2}-I(v) \geq 0$, consequently, $b_{2} \geq \hat{C}$.

$b_{2} \leq b_{1}$. For $u \in \mathcal{N}^{+}$, there exists $s_{0}>1$ such that $I\left(t_{0} u\right)<0$. Set $h(s):=\bar{I}\left(s s_{0} u\right)$, similar to Lemma 3.7, we know that $h(0)=0, h(s)>0$ for $s$ sufficiently small, and $h(s) \rightarrow-\infty$ as $s \rightarrow \infty$. Thus there is $s\left(s_{0} u\right)>0$ such that $I\left(s\left(s_{0} u\right) s_{0} u\right)=\sup _{t \geq 0} I\left(s s_{0} u\right)$, so $s\left(s_{0} u\right) s_{0} u \in \mathcal{N}^{+}$and $I^{\prime}\left(s\left(s_{0} u\right) s_{0} u\right)=$ 0 . Let $\gamma(s)=s s_{0} u$, then $\gamma(s) \in \mathcal{M}$ and

$$
b_{2} \leq \max _{s \in[0,1]} I(\gamma(s))=I\left(s\left(s_{0} u\right) s_{0} u\right) .
$$

This complete the proof of the lemma.

Let $u_{0} \in E^{+}$be such that $I\left(u_{0}\right)<0$, and set

$$
\begin{aligned}
\mathcal{M}_{0} & :=\left\{\gamma \in C\left([0,1], E^{+}\right): \gamma(0)=0, \gamma(1)=u_{0}\right\} . \\
b_{0} & :=\inf _{\gamma \in \mathcal{M}_{0}} \sup _{s \in[0,1]} I(\gamma(s)) .
\end{aligned}
$$

Lemma 3.10. There holds $b_{0}=b_{2}$.

Proof. Since $\mathcal{M}_{0} \subset \mathcal{M}$, it is clear that $b_{2} \leq b_{0}$. Let $\gamma \in \mathcal{M}$. Then as before $I(s \gamma(1))$ and $I\left(s u_{0}\right)$ are strictly decreasing for $s \geq 1$, and $I(s \gamma(1)) \rightarrow-\infty$, $I\left(s u_{0}\right) \rightarrow-\infty$ as $s \rightarrow \infty$. Let $\nu(s)$ be a curve in the two-dimensional subspace $\operatorname{span}\left\{\gamma(1), u_{0}\right\}$ jointing $\gamma(1)$ and $u_{0}$ such that $I(\nu(s))<0$ for $1 \leq s \leq 2$ (such a curve exists because of the conclusion (2) of Lemma 3.7). Define $\hat{\nu}(s)$ by $\hat{\nu}(s)=\gamma(2 s)$ for $s \in[0,1 / 2]$ and $\hat{\nu}(s)=\nu(2 s)$ for $s \in[1 / 2,1]$. Then $\hat{\nu}(s) \in \mathcal{M}_{0}$ and $\max _{s \in[0,1]} I(\hat{\nu}(s))=\max _{s \in[0,1]} I(\hat{\nu}(s))$. Thus $b_{0} \leq b_{2}$. 
Lemma 3.11. Let $u \in \mathcal{K}^{+}$be such that $I(u)=\hat{C}$, and set $E_{u}=E^{-} \oplus \mathbb{R} u$. Then

$$
\sup _{w \in E_{u}} F(w)=I(u) \text {. }
$$

Proof. For any $w=v+s u \in E_{u}$, by (3.5),

$$
\begin{aligned}
F(w) & =\frac{1}{2}\|s u\|^{2}-\frac{1}{2}\|v\|^{2}-\int_{{\mathbb{R} \times \mathbb{R}^{N}}_{\infty}} H_{\infty}^{\prime}(v+s u) \\
& \leq F(s u+f(s u))=I(s u) .
\end{aligned}
$$

Since $u \in \mathcal{N}^{+}$, one has

$$
\sup _{w \in E_{u}} F(w) \leq \sup _{s \geq 0} I(s u)=I(u)
$$

\section{Proof of Theorem 1.1}

We are now going to complete the proof of the main result. Let $\hat{\mathcal{K}}:=\{u \in E$ : $\left.\Phi^{\prime}(u)=0\right\}$ be the critical set of $\Phi$. Recall that

$$
c_{\text {min }}:=\inf \{\Phi(u): u \in \hat{\mathcal{K}}\}
$$

and

$$
S_{\text {min }}:=\inf \left\{u \in \hat{\mathcal{K}}: \Phi(u)=c_{\text {min }}\right\} .
$$

From the conditions $\left(\mathcal{H}_{1}\right)-\left(\mathcal{H}_{4}\right)$, it yields that for any $\varepsilon>0$, there is $C_{\varepsilon}>0$ such that

$$
\left|H_{z}(\hat{x}, z)\right| \leq \varepsilon|z|+C_{\varepsilon}|z|^{p-1}
$$

and

$$
|H(\hat{x}, z)| \leq \varepsilon|z|^{2}+C_{\varepsilon}|z|^{p}
$$

for all $(\hat{x}, z)$, where $\frac{2 \mu}{\mu-1} \leq p<N^{*}=\frac{2(N+2)}{N}$. we have

Now we study the functional $\Phi$ defined by (2.2). From Lemma 4.1 of [16],

Lemma 4.1. $\Psi$ is nonnegative and weakly sequentially lower semicontinuous and $\Phi^{\prime}$ is weakly sequentially continuous.

Lemma 4.2. There exist $\kappa>0$ and $\rho>0$ such that $\left.\Phi\right|_{M_{\rho}^{+}} \geq 0$ and $\left.\Phi\right|_{\partial M_{\rho}^{+}} \geq \kappa$ where $M_{\rho}^{+}:=\left\{u \in E^{+}:\|u\| \leq \rho\right\}$ and $\partial M_{\rho}^{+}:=\left\{u \in E^{+}:\|u\|=\rho\right\}$.

Proof. By (4.2) and (2.1), for $u \in E^{+}$one has

$$
\begin{aligned}
\Phi(u) & =\frac{1}{2}\|u\|^{2}-\frac{1}{2} \int_{\mathbb{R} \times \mathbb{R}^{N}} B_{L} z \cdot z-\int_{\mathbb{R} \times \mathbb{R}^{N}} H(\hat{x}, u) \\
& \geq \frac{1}{2}\|u\|^{2}-\frac{\lambda}{2}|z|_{2}^{2}-\varepsilon|u|_{2}^{2}-C_{\varepsilon}|u|_{p}^{p} \\
& \geq\left(\frac{1}{2}-\frac{\lambda}{2\left(V_{0}-\mu_{\infty}\right)}\right)\|u\|^{2}-\varepsilon|u|_{2}^{2}-C_{\varepsilon}|u|_{p}^{p},
\end{aligned}
$$


where $\lambda>0$ is defined in $\left(\mathcal{H}_{0}\right)$. Now the desired conclusion follows from Lemma 2.6 and $\left(\mathcal{H}_{0}\right)$.

Lemma 4.3. There is $R>0$ such that, for any $e \in E^{+}$with $\|e\|=1$ and $E_{e}=E \oplus \mathbb{R} e$,

$$
\Phi(u)<0, \quad \forall u \in E_{e} \backslash K_{R}
$$

where $K_{R}:=\left\{u \in E_{e}:\|u\| \leq R\right\}$.

Proof. For $z \in E_{e}$, we have

$$
\begin{aligned}
\Phi(z) & =\frac{1}{2}\left\|z^{+}\right\|^{2}-\frac{1}{2}\left\|z^{-}\right\|^{2}-\frac{1}{2} \int_{\mathbb{R} \times \mathbb{R}^{N}} B_{L} z \cdot z-\int_{\mathbb{R} \times \mathbb{R}^{N}} H(\hat{x}, z) \\
& \leq \frac{1}{2}\left\|z^{+}\right\|^{2}-\frac{1}{2}\left\|z^{-}\right\|^{2}-\int_{\mathbb{R}^{2} \mathbb{R}^{N}} H_{\infty}(z)=F(z),
\end{aligned}
$$

Thus, this lemma follows easily from Lemma 3.2.

In particular, let $U \in \mathcal{K}$ with $F(U)=\hat{C}$. Set $e \equiv U^{+}$and $E_{e}=E \oplus \mathbb{R} e$.

Lemma 4.4. We have

$$
d:=\sup \left\{\Phi(u): u \in E_{e}\right\}<\hat{C} .
$$

Proof. Observe that by Lemma 4.2 and the linking property we have $d \geq \kappa$.

By $\left(\mathcal{H}_{4}\right)$, we see that $\Phi(u) \leq F(u)$ for all $u=v+s U^{+}$, and

$$
F(u)=F\left(v+s U^{+}\right) \leq F\left(s U^{+}+f\left(s U^{+}\right)\right) \leq \hat{C},
$$

hence $d \leq \hat{C}$. Assume by contradiction that $d=\hat{C}$. Let $w_{j}:=v_{j}+s_{j} U^{+} \in E_{e}$ be such that $d-\frac{1}{j} \leq \Phi\left(w_{j}\right) \rightarrow d$. It follows from Lemma 4.3 that $w_{j}$ is bounded and we can assume $w_{j} \rightarrow w$ in E with $v_{j} \rightarrow v$ and $s_{j} \rightarrow s$. It is clear that $s>0$ (otherwise there should appear the contradiction that $d=0$ ). Then

$$
d-\frac{1}{j} \leq \Phi\left(w_{j}\right) \leq F\left(w_{j}\right)-\frac{1}{2} \int_{\mathbb{R} \times \mathbb{R}^{N}} B_{L} w_{j} \cdot w_{j} \leq \hat{C}
$$

Taking the limit yields $\hat{C} \leq \hat{C}-\frac{1}{2} \int_{\mathbb{R} \times \mathbb{R}^{N}} B_{L} w \cdot w$ which implies that $w=0$, a contradiction.

Set

$$
Q:=\left\{u=u^{-}+s U^{+}: u^{-} \in E^{-}, s \geq 0,\|u\|<R\right\}
$$

As a consequence of Lemma 4.4 one has the following

Lemma 4.5. $\sup \Phi(Q)<\hat{C}$.

We now turn to the analysis on $(C)_{c}$-sequences. First we have

Lemma 4.6. Any $(C)_{c}$-sequence for $\Phi$ is bounded.

Proof. The proof was given in Lemmas 5.1-5.3 of [16].

Lemma 4.7. Let $\left\{z_{n}\right\} \subset E$ be a $(C)_{c}$-sequence. Then either

(i) $z_{n} \rightarrow z_{L}$, or 
(ii) $c \geq \hat{C}$ and there exists a positive integer $\ell \leq\left[\frac{c}{\hat{C}}\right]$, points $\bar{z}_{1}, \ldots, \bar{z}_{\ell} \in$ $\mathcal{K} \backslash\{0\}$ (not necessarily distinct), a subsequence of denote again by $\left\{z_{n}\right\}$ and sequence $\left\{k_{n}^{i}\right\} \subset \mathbb{Z}^{N+1}(i=1, \ldots, \ell)$ such that

$\left\|z_{n}-z_{L}-\Sigma_{i=1}^{\ell} k_{n}^{i} * \bar{z}_{i}\right\|_{\nu} \rightarrow 0, \quad\left|k_{n}^{i}-k_{n}^{j}\right| \rightarrow \infty(i \neq j)$ as $n \rightarrow \infty$,

and

$$
\Phi\left(z_{L}\right)+\Sigma_{i=1}^{\ell} F\left(\bar{z}_{i}\right)=c
$$

Proof. Remark that, by Lemma 4.6 any $(C)_{c}$-sequence is bounded, hence it is a $(P S)_{c}$-sequence. The proof is now well known, see for example Alama and $\mathrm{Li}[2]$ (see also Lemma 4.8 of [15] or Lemma 4.7 of [14]). Here we outline it as follows.

First observe that $c \geq 0$ which follows by taking the limit in

$$
\Phi\left(z_{n}\right)-\frac{1}{2} \Phi^{\prime}\left(z_{n}\right) z_{n}=\int_{\mathbb{R} \times \mathbb{R}^{N}} \tilde{H}(\hat{x}, z) \geq 0 .
$$

Assume $(i)$ is false. It is easy to see that $z_{n}^{1}:=z_{n}-z_{L}$ is a $(P S)_{c_{1}}$ sequence for $F$ with $c_{1}=c-\Phi\left(z_{n}\right)$ and $z_{n}^{1} \rightarrow 0$. Note that $F$ is invariant under the $*$-action of $\mathbb{Z}^{N+1}$. A standard argument of concentration compactness principle implies that there exist a sequence $k_{n}^{1} \in \mathbb{Z}^{N+1}$ with $\left|k_{n}^{1}\right| \rightarrow \infty$ and a critical point $\bar{z}_{1} \neq 0$ of $F$ satisfying $k_{n}^{1} * z_{n}^{1} \rightarrow \bar{z}_{1}$ and

$$
F\left(k_{n}^{1} * z_{n}^{1}\right) \rightarrow c-\Phi\left(z_{L}\right)-F\left(\bar{z}_{1}\right) \geq 0
$$

Since $\Phi\left(z_{L}\right) \geq 0$ and $F\left(\bar{z}_{1}\right) \geq \hat{C}$, one sees that $c \geq \hat{C}$.

If $k_{n}^{1} * z_{n}^{1} \rightarrow \bar{z}_{1}$ then we are done. Otherwise, repeating the above argument, after at most finitely many steps we finish the proof.

Proof of Theorem 1.1. We first prove our existence results. From the form of $\Phi$ (see $(2.4)$ ) and $H(\hat{x}, z) \geq 0$, we see that $\Phi$ satisfies $\left(\mathcal{A}_{0}\right)$. The combination of Lemmas 4.1 and Theorem 2.10 implies that $\Phi$ verifies $\left(\mathcal{A}_{1}\right)$. Lemma 4.2 implies that $\left(\mathcal{A}_{2}\right)$ holds, and Lemma 4.3 shows that the linking condition of Theorem 2.9 is satisfied. These together with Lemma 4.5 imply that $\Phi$ has a

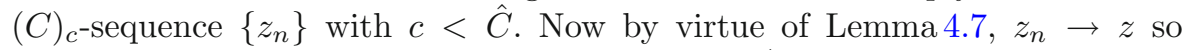
that $\Phi^{\prime}(z)=0$ and $\Phi(z) \geq \kappa$. This proves that $\hat{\mathcal{K}} \backslash\{0\} \neq \varnothing$. By Lemma 2.8, $\hat{\mathcal{K}} \subset \cap_{r \geq 2} B_{r}$.

As in the proof of Lemma 3.4 one has $c_{\text {min }} \geq 0$. Let $\left\{u_{j}\right\} \subset \hat{\mathcal{K}} \backslash\{0\}$ such that $\Phi\left(u_{j}\right) \rightarrow c_{m i n}$. Then $\left\{u_{j}\right\}$ is a $(C)_{c_{m i n}}$-sequence, and since $c_{m i n}<\hat{C}$, then Lemma 4.7 implies $u_{j} \rightarrow u \in S_{\text {min }}$. Note that $\Phi\left(u_{j}\right)=\int_{\mathbb{R}^{\prime} \mathbb{R}^{N}} \tilde{H}\left(\hat{x}, u_{j}\right) \rightarrow c_{m i n}$, and by assumptions it holds that, for any $\varepsilon>0$, there is $C_{\varepsilon}>0$ with

$$
\frac{\left|H_{z}(\hat{x}, u)\right|}{|u|} \leq \varepsilon+C_{\varepsilon} \tilde{H}_{z}(\hat{x}, u)^{\frac{1}{\mu}} .
$$


Using the representation (2.4) and the estimate (2.3) we get

$$
\begin{aligned}
\left\|u_{j}\right\|_{B}^{2} & =\int_{\mathbb{R} \times \mathbb{R}^{N}} H_{z}\left(\hat{x}, u_{j}\right)\left(u_{j}^{+}-u_{j}^{-}\right) \\
& \leq \varepsilon\left|u_{j}\right|_{2}^{2}+C_{\varepsilon}\left(\int_{\mathbb{R}^{\prime} \mathbb{R}^{N}} \tilde{H}\left(\hat{x}, u_{j}\right)\right)^{\frac{1}{\mu}}\left|u_{j}\right|_{p}\left(\int_{\left.{\mathbb{R} \times \mathbb{R}^{N}}\left|u_{j}^{+}-u_{j}^{-}\right|^{p}\right)^{\frac{1}{p}}}\right. \\
& \leq c \varepsilon\left\|u_{j}\right\|_{B}^{2}+c_{1} C_{\varepsilon}\left(\Phi\left(u_{j}\right)\right)^{\frac{1}{\sigma}}\left\|u_{j}\right\|_{B}^{2}
\end{aligned}
$$

where $p=\frac{2 \mu}{\mu-1}$. Hence $1 \leq c \varepsilon+c_{1} C_{\varepsilon} c_{\text {min }}^{\frac{1}{\mu}}$, consequently $c_{\text {min }}>0$.

We now prove that $S_{\text {min }}$ is compact in $B_{2}$. Note that, by $\left(\mathcal{H}_{0}\right)-\left(\mathcal{H}_{4}\right)$, it is not difficult to show that $S_{\min }$ is bounded in E (cf. [16] or [18]), hence, $|u|_{2} \leq C_{2}$ for all $u \in S_{\min }$ and some $C_{2}$. Therefore, as a consequence of Lemma 2.8 we see that, for each $q \in[2, \infty)$, there is $\Lambda_{q}>0$ such that

$$
\|u\|_{B_{q}} \leq \Lambda_{q} \text { for all } u \in S_{\text {min }} .
$$

This together with the Sobolev embedding theorem (see [5]) implies that for some $\Lambda_{\infty}$ independent of $u \in S_{\min }$ satisfying

$$
|u|_{\infty} \leq \Lambda_{\infty} \text { for all } u \in S_{\text {min }} .
$$

Let $u_{j} \in S_{\min }$ be such that $u_{j} \rightarrow u$ in $B_{2}$. Since $\Phi\left(u_{j}\right)=c_{\min }$ and $\Phi^{\prime}\left(u_{j}\right)=0$,

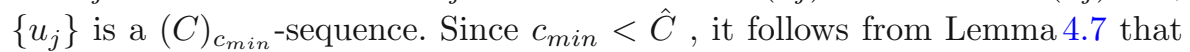
$u_{j} \rightarrow u$ (along a subsequence) in E. Clearly, $u \in S_{\text {min }}$. By

$$
L_{\infty} u=B_{L} u+H_{z}(\hat{x}, u)
$$

one has

$$
\begin{aligned}
\left|L_{\infty}\left(u_{j}-u\right)\right|_{2} & \leq\left|B_{L}\left(u_{j}-u\right)\right|_{2}+\left|H_{z}\left(\cdot, u_{j}\right)-H_{z}(\cdot, u)\right|_{2} \\
& \leq o(1)+\left|H_{z}\left(\cdot, u_{j}\right)-H_{z}(\cdot, u)\right|_{2} .
\end{aligned}
$$

Since $\left|u_{j}\right|_{\infty} \leq \Lambda_{\infty}, u_{j} \rightarrow u$ in $\mathrm{E}$ and $|u(\hat{x})| \rightarrow 0$ as $|\hat{x}| \rightarrow \infty$, then we have

$$
\left|H_{z}\left(\cdot, u_{j}\right)-H_{z}(\cdot, u)\right|_{2} \rightarrow 0 .
$$

Therefore, one sees that $\left|L_{\infty}\left(u_{j}-u\right)\right|_{2} \rightarrow 0$, i.e., $u_{j} \rightarrow u$ in $B_{2}$.

\section{Acknowledgements}

Many thanks to the referees for careful reading and helpful suggestions which make us possible to refine the paper.

\section{References}

[1] Ackermann, N.: A nonlinear superposition principle and multibump solutions of periodic Schröinger equations. J. Funct. Anal. 234, 423-443 (2006)

[2] Alama, A., Li, Y.Y.: On multibump bound states for certain semilinear elliptic equations. Indiana Univ. Math. J. 41, 983-1026 (1992) 
[3] Bartsch, T., Ding, Y.H.: Deformation theorems on non-metrizable vector spaces and applications to critical point theory. Math. Nachr. 279, 1267-1288 (2006)

[4] Bartsch, T., Ding, Y.H.: Homoclinic solutions of an infinite-dimensional Hamiltonian system. Math. Z. 240, 289-310 (2002)

[5] Besov, O., Ilín, V., Nikolśkii, S.: Integral Representations of Functions and Embedding Theorems (Russian). Izd. Nauka, Moscow (1975)

[6] Bartsch, T., Ding, Y.H.: On a nonlinear Schrödinger equations. Math. Ann. 313, 15-37 (1999)

[7] Barbu, V.: Periodic solutions to unbounded Hamiltonian system. Discrete Contin. Dyn. Syst. 1, 277-283 (1995)

[8] Brézis, H., Nirenberg, L.: Characterization of the ranges of some nonlinear operators and applications to boundary value problems. Ann. Scuola Norm. Sup. Pisa Cl. Sci. 5, 225-326 (1978)

[9] Bartsch, T., De Figueiredo, D.G.: Infinitely many solutions of nonlinear elliptic systems. In: Progress in Nonlinear Differential Equations and Their Applications, vol. 35, pp. 51-67. Birkhöauser, Basel (1999)

[10] Clément, P., Van der Vorst, R.C.A.M.: On the non-existence of homoclinic orbits for a class of infinite dimensional Hamiltonian systems. Proc. Am. Math. Soc. 125, 1167-1176 (1997)

[11] Clément, P., Felmer, P., Mitidieri, E.: Homoclinic orbits for a class of infinite dimensional Hamiltonian systems. Ann. Scuola Norm. Sup. Pisa Cl. Sci. 24, 367393 (1997)

[12] Clément, P., De Figueiredo, D.G., Mitidieri, E.: Positive solutions of semilinear elliptic systems. Comm. Partial Differ. Equ. 17, 923-940 (1992)

[13] Clément, P., Van der Vorst, R.C.A.M.: On a semilinear elliptic system. Differ. Integral Equ. 8, 1317-1329 (1995)

[14] Ding, Y.H., Lee, C.: Existence and exponential decay of homoclinics in a nonperiodic superquadrtic Hamiltonian system. J. Differ. Equ. 246, 2829-2848 (2009)

[15] Ding, Y.H., Wei, J.C.: Stationary state of nonlinear Dirac equations with general potentials. Rev. Math. Phys. 20(8), 1007-1032 (2008)

[16] Ding, Y.H., Luan, S.X., Willem, M.: Solutions of a system of diffusion equations. J. Fixed Point Theory Appl. 2, 117-139 (2007)

[17] Ding, Y.H., Lee, C.: Periodic solutions of an infinite-dimensional Hamiltonian system. Rocky Mt. J. Math. 35, 1881-1908 (2005)

[18] Ding, Y.H.: Variational Methods for Strongly Indefinite Problems. World Scientific Press, Singapore (2008)

[19] De Figueiredo, D.G., Felmer, P.L.: On superquadratic elliptic systems. Tran. Am. Math. Soc. 343, 99-116 (1994) 
[20] De Figueiredo, D.G., Ding, Y.H.: Strongly indefinite functionals and multiple solutions of elliptic systems. Tran. Am. Math. Soc. 355, 2973-2989 (2003)

[21] De Figueiredo, D.G., do Ó, J.M., Ruf, B.: An Orlicz-space approach to superlinear elliptic systems. J. Funct. Anal. 224, 471-496 (2005)

[22] Esteban, M., Séré, E.: Stationary states of the nonlinear Dirac equation: A variational approach. Comm. Math. Phys. 171, 250-323 (1995)

[23] Hulshof, J., Van der Vorst, R.C.A.M.: Differential systems with strongly indefinite structure. J. Funct. Anal. 114, 32-58 (1993)

[24] Kryszewski, W., Szulkin, A.: An infinite dimensional Morse theory with applications. Tran. Am. Math. Soc. 349, 3181-3234 (1997)

[25] Kryszewski, W., Szulkin, A.: Generalized linking theorem with an application to semi-linear Schrödinger equation. Adv. Differ. Equ. 3, 441-472 (1998)

[26] Lions, P.L.: The concentration-compactness principle in the calculus of variations: The locally compact cases, part II. Ann. Inst. H. Poincaré Anal. Non Linéaire 1, 223-283 (1984)

[27] Lions, J.L.: Optimal Control of Systems Governed by Partial Differential Equations. Springer, New York (1971)

[28] Mitidieri, E.: A Rellich type identy and applications. Comm. Partial Differ. Equ. 17, 125-151 (1993)

[29] Mitidieri, E., Hulshof, J., Van der Vorst, R.C.A.M.: Strongly indefinite systems with critical Sobolev exponents. Trans. Am. Math. Soc. 350, 2349-2365 (1998)

[30] Nagasawa, M.: Schrödinger Equations and Diffusion Theroy. Birkhäuser, Basel (1993)

[31] Schechter, M., Zou, W.: Homoclinic orbits for Schrödinger systems. Mich. Math. J. 51, 59-71 (2003)

[32] Zhao, F.K., Ding, Y.H.: On a diffusion system with bounded potential. Discrete Contin. Dyn. Syst. 23, 1073-1086 (2009)

J. Wang, J. Xu, F. Zhang, L. Wang

Department of Mathematics, Southeast University,

Nanjing 210096, People's Republic of China

e-mail: wangjdn2006@21cn.com

J. Xu

e-mail: xujun@seu.edu.cn

F. Zhang

e-mail: zhangfubao@seu.edu.cn

Received: 08 May 2009.

Accepted: 27 January 2010. 